\title{
Vertical sorting and the morphodynamics of bed-form-dominated rivers: An equilibrium sorting model
}

\author{
Astrid Blom \\ Water Engineering and Management, Civil Engineering, University of Twente, Netherlands \\ Gary Parker \\ Department of Civil and Environmental Engineering and Department of Geology, University of Illinois at Urbana- \\ Champaign, Urbana, Illinois, USA
}

Jan S. Ribberink and Huib J. de Vriend

Water Engineering and Management, Civil Engineering, University of Twente, Netherlands

Received 7 June 2004; revised 22 September 2005; accepted 4 October 2005; published 9 February 2006.

[1] A modeling framework is developed for taking into account the effects of sediment sorting in the morphodynamic modeling of bed-form-dominated rivers for the case of equilibrium or stationary conditions dominated by bed load transport. To this end, the Blom and Parker (2004) framework for sediment continuity is reduced to an equilibrium sorting model. The predicted equilibrium sorting profile is mainly determined by the probability density function (PDF) of bed form trough elevations and by a lee sorting function. The PDF of trough elevations needs to be known from either model predictions or measurements. A simple formulation for the lee sorting function is suggested, yet data on the avalanche mechanism down lee faces of dunes is required so as to improve the function and make it generic. The equilibrium sorting model is calibrated and verified using data from flume experiments. The agreement between the predicted and measured equilibrium sorting profiles is reasonable, although the model does not reproduce an observed coarse top layer. In a hydraulic-morphodynamic model this equilibrium sorting model may be applied instantaneously if the timescale of large-scale morphological changes is much larger than the ones of changes in vertical sorting and dune dimensions.

Citation: Blom, A., G. Parker, J. S. Ribberink, and H. J. de Vriend (2006), Vertical sorting and the morphodynamics of bed-formdominated rivers: An equilibrium sorting model, J. Geophys. Res., 111, F01006, doi:10.1029/2004JF000175.

\section{Introduction}

[2] Morphodynamic model systems are important tools in the analysis and prediction of the effects of river interventions or, for instance, the river's hydraulic and morphodynamic response to a flood event. A morphodynamic model system is here defined as a system that couples modules for calculating flow, sediment transport, and net aggradation or degradation of the river bed. Examples are the Delft3D model system of WL Delft Hydraulics and the MIKE model series of the Danish Hydraulics Institute. In case sediment sorting processes play a role, the river bed material cannot be characterized by a single grain size, and the sediment continuity model applied in the morphodynamic model system needs to describe the interaction among grain-sizeselective sediment transport, net aggradation or degradation, and the vertical sorting profile. Hirano [1971] was the first to develop such a sediment continuity model for nonuniform sediment.

Copyright 2006 by the American Geophysical Union. 0148-0227/06/2004JF000175\$09.00
[3] Yet this commonly used Hirano active layer model and its variants suffer from three main shortcomings [Blom and Parker, 2004]. First, in most of the Hirano type bed layer models vertical sediment fluxes occur through net aggradation or degradation only, whereas flume experiments have shown that these fluxes also occur in situations without net aggradation or degradation. Secondly, in certain situations the set of equations of sediment continuity models with discrete bed layers becomes elliptic in parts of the space-time domain. Solving the set of equations then requires future time boundaries, which is physically unrealistic. Finally, from a physical point of view it is not straightforward to distinguish between the range of bed elevations interacting with the flow regularly (i.e., the active layer) and the range not interacting with the flow, at all (i.e., the substrate).

[4] In reality, the active part of the bed does not constitute a distinct surface layer as proposed by Hirano, but it is rather represented by a probability density function (PDF) of bed surface elevations. The depth-continuous framework for sediment continuity developed by Parker et al. [2000] (i.e., the Parker-Paola-Leclair framework for sediment con- 
tinuity, or PPL framework) is particularly adequate for this purpose. It allows us to take into account that relatively low bed elevations interact with the flow and are subject to entrainment and deposition less frequently than higher ones.

[5] The study by Blom and Parker [2004] encompasses the derivation of formulations for the grain-size-specific and bed elevation-specific entrainment and deposition fluxes as required for the PPL framework. As such, the Blom-Parker framework (or BP framework) describes the bed composition and vertical sorting fluxes without distinguishing discrete bed layers, and is aimed at conditions dominated by bed forms (river dunes) and bed load transport. The BP framework will be explained in further detail in the next section.

[6] The BP framework would be complete using a submodel for the size-selective entrainment over bed forms. As such a model is not readily available, we take another approach in the present paper. We will reduce the BP framework to an equilibrium sorting model for the case of equilibrium conditions $(\partial / \partial t=0)$. Equilibrium is here defined as the situation in which all parameters, e.g., grain-size-specific transport rates and the mean bed level, vary around mean values. In a follow-up paper, we will reduce the BP framework to a sorting evolution model.

[7] The present paper starts with an explanation of the BP framework (section 2). As a first step toward nonuniform sediment, we will consider uniform sediment (section 3 ). We then consider tracer particles in uniform sediment (section 4), and finally nonuniform sediment (section 5). The equilibrium sorting model will be calibrated in section 7 using data from flume experiments conducted by Blom et al. [2003]. The model is verified using data from flume experiments conducted by Ribberink [1987] (section 8). As the present paper is a follow-up of the paper by Blom and Parker [2004], the same notation will be used. Reference to equations of Blom and Parker [2004] will be specified by (BP-equation number).

\section{Blom-Parker (BP) Framework}

[8] Blom and Parker [2004] have developed a new continuum sorting model, applicable to bed-form-dominated rivers, which is based on (1) the PPL framework for sediment continuity [Parker et al., 2000], (2) the Einstein step length formulation [Einstein, 1950], (3) a lee sorting function, and (4) a method to account for the variability in bed form dimensions. These four elements will be shortly explained below.

[9] The PPL framework for sediment continuity does not distinguish between discrete bed layers and is based on a PDF of bed surface elevations of a bed form covered bed. This PDF indicates the likelihood of a certain bed elevation being exposed to the flow. As such, the framework enables relating vertical sorting fluxes to the likelihood of a certain bed elevation being exposed to the flow. Appendix A presents the fundamental equations of the PPL framework.

[10] Each bed form is divided into a stoss and a leeside (Figure 1). It is assumed that on the stoss side deposition and entrainment occur simultaneously, while on the leeside only deposition occurs. The step length formulation first introduced by Einstein [1950] is applied to the stoss face. The Einstein step length is defined as the average distance

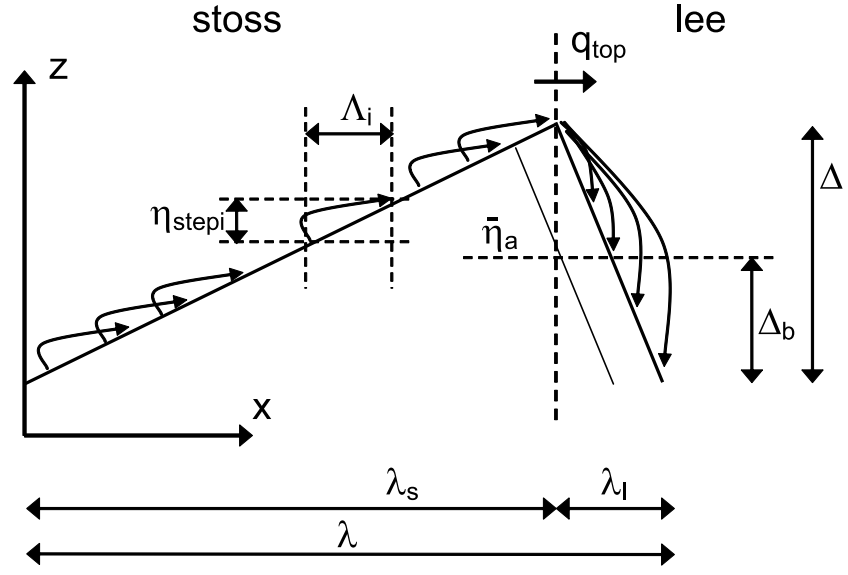

Figure 1. Bed form parameters and division of bed form in stoss and leesides with accompanying entrainment and deposition fluxes.

covered by a particle from the moment it is picked up until saltation ceases and a period of rest on the bed begins. Einstein [1950] proposes that the average step length, $\Lambda$, is a linear function of the grain diameter, $d(\Lambda=\alpha d$ where $\alpha$ denotes the dimensionless step length).

[11] In order to incorporate sorting through the avalanche mechanism at the lee face, a simple lee sorting function was developed describing the grain-size-selective deposition rate down the avalanche lee face of a bed form. Two constants in the lee sorting function represent the relative importance of the relative grain size and the dimensionless bed shear stress, respectively. In the present paper these constants will be used as calibration coefficients. The lee sorting function is a simple trend-based model, and it seems to be the simplest form that has any hope of capturing the effects in question.

[12] The variation in bed form dimensions has been taken into account by incorporating the statistics of the trough elevations. The geometric properties of an individual bed form (e.g., dune height $\Delta$ and dune length $\lambda$ ) are assumed to be related to the relative trough elevation $\Delta_{b}$ (see Figure 1) according to simple relations. All bed forms in the series are assumed to have a triangular shape. As such, the likelihood of occurrence of a specific bed form is characterized by the PDF of relative trough elevations.

[13] Appendix B lists the main equations of the BP framework.

\section{Uniform Sediment}

[14] As a first step toward nonuniform sediment, we consider a series of irregular bed forms migrating over a bed composed of uniform sediment. For uniform sediment in equilibrium conditions $(\partial / \partial t=0)$, sediment conservation equations (A3) and (A4) reduce to

$$
\begin{aligned}
0= & \bar{D}_{e s E}(z)-\bar{E}_{e s E}(z)+\bar{D}_{\text {elE }}(z) \\
= & \int_{\eta_{b \min }}^{\eta_{b \max }}\left[\frac{\lambda_{s}}{\lambda} p_{s e}(z) E_{s u E}\left(z-\eta_{s t e p}(z)\right)-\frac{\lambda_{s}}{\lambda} p_{s e}(z) E_{s u E}(z)\right. \\
& \left.+\frac{\lambda_{l}}{\lambda} p_{l e}(z) D_{l E}\right] \tilde{p}_{b E} d \eta_{b}
\end{aligned}
$$


using expressions for entrainment and deposition densities (B5)-(B7). The subscript $E$ indicates equilibrium conditions. The various parameters have been defined in Appendices A and B. In order to find the elevationspecific deposition and entrainment fluxes averaged over a series of irregular bed forms, $\bar{D}_{e s E}, \bar{E}_{e s E}$, and $\bar{D}_{e l E}$, we need to solve for the entrainment rate $E_{s u E}$ over individual bed forms.

[15] Equation (1) is further reduced by assuming that not only each individual lee face has a uniform slope, but also each stoss face $\left(p_{s e}(z)=p_{l e}(z)=J(z) / \Delta\right)$. Note, however, that the slope of the stoss face is not equal to the slope of the lee face. Thus we have simplified the series of irregular bed forms to a series of triangular bed forms with varying dune heights and dune lengths. Now equation (1) yields

$$
\begin{aligned}
0= & \int_{\eta_{b \min }}^{\eta_{b \max }} \frac{J(z)}{\lambda \Delta}\left[\lambda_{s} E_{\text {suE }}\left(z-\eta_{\text {step }}(z)\right)-\lambda_{s} E_{\text {suE }}(z)+q_{t o p E}\right] \\
& \cdot \tilde{p}_{b E} d \eta_{b}
\end{aligned}
$$

since in equilibrium conditions the deposition rate at the lee face $D_{l E}$ (equation (B8)) reduces to $q_{t o p E} / \lambda_{l}$. Equation (2) expresses the following: in equilibrium conditions at each bed elevation $z$ there is a dynamic equilibrium between (1) the net entrainment flux (i.e., the difference between the entrainment flux and the deposition flux) at the stoss face, averaged over all bed forms and (2) the deposition flux at the lee face, averaged over all bed forms. This is illustrated in Figure 2a. We now assume that, for each individual dune in a series of irregular dunes, the net entrainment flux at bed elevation $z$ at the stoss face equals the deposition rate at the lee face:

$$
\lambda_{s} E_{s u E}\left(z-\eta_{\text {step }}(z)\right)-\lambda_{s} E_{\text {suE }}(z)+q_{\text {top E }}=0
$$

whence the constraint of equation (2) is satisfied. This means that the model does not allow dune migration to change the shape of individual dunes. Note, however, that equation (2) might also be met for different distributions of the different quantities over $z$. Yet the assumption is made in order to find a solution to the model and to push the model forward.

[16] Now, in order to find a solution to $E_{s u E}(z)$ that fulfills equation (3), we first have a look at the formulation for the bed load transport rate over the bed form crest, $q_{\text {topE }}$. For nonuniform sediment, the transport rate over an individual bed form crest is given by equation (B11). Similar to equation (B4), the bed load transport rate over the bed form crest for uniform sediment is given by

$$
q_{\text {top } E}=\Lambda E_{\text {suE }}\left(z_{t}-\frac{1}{2} \eta_{\text {step }}\left(z_{t}\right)\right)
$$

where $z_{t}$ denotes the crest elevation of the specific bed form $\left(z_{t}=\eta_{t}=\bar{\eta}_{a}+\frac{1}{2} \Delta\right)$. Based on the following equation, which translates the step length $\Lambda_{i}$ into the dimensionless vertical step length at elevation $z$ on the stoss face for size fraction $i$, $\eta_{\text {stepi }}^{*}$

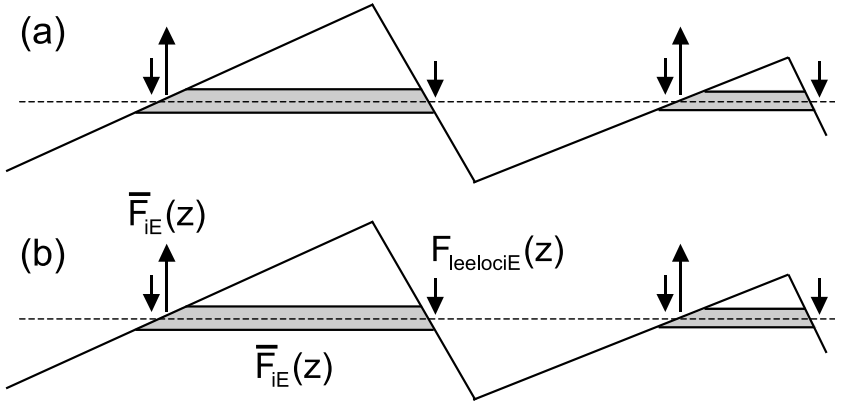

Figure 2. (a) Sediment fluxes onto bed elevation $z$ for a series of irregular triangular bed forms and (b) composition of sediment fluxes onto elevation $z$.

(equal to equation (BP-B14) where $\eta_{\text {stepi }}^{*}=\eta_{\text {stepi }} / \Delta$ and $\Lambda_{i}^{*}=\Lambda_{i}\left(\lambda_{s}\right)$, the arguments in equations (3) and (4) can be written as

$$
\begin{aligned}
& z-\eta_{\text {step }}(z)=\Delta f_{d s}\left[g_{d s}\left(z^{*}\right)-\Lambda / \lambda_{s}\right]+\eta_{a} \\
& z_{t}-\frac{1}{2} \eta_{\text {step }}\left(z_{t}\right)=\Delta f_{d s}\left[1-\Lambda /\left(2 \lambda_{s}\right)\right]+\eta_{a}
\end{aligned}
$$

where the function $f_{d s}$ expresses the dimensionless elevation of the stoss face as a function of the dimensionless horizontal coordinate, and $g_{d s}$ is its inverse:

$$
\begin{aligned}
& f_{d s}\left(x_{s}^{*}\right)=x_{s}^{*}-\frac{1}{2} \\
& g_{d s}\left(z^{*}\right)=z^{*}+\frac{1}{2}
\end{aligned}
$$

also see equations (BP-B10) and (BP-B11). Herein $x_{s}^{*}=x /$ $\lambda_{s}$ and $z^{*}=\left(z-\eta_{a}\right) / \Delta$.

[17] Analyzing equations (3)-(7), it is found that a possible solution to the entrainment rate over an individual bed form, $E_{s u E}$, is a linear function of $g_{d s}$ :

$$
E_{\text {suE }}(z)=a_{E} g_{d s}\left(z^{*}\right)+b_{E}
$$

where $a_{E}$ and $b_{E}$ are constants, independent of $z$. This implies that for a triangular bed form the entrainment rate $E_{s u E}$ increases linearly with increasing bed surface elevation. This can also be understood by applying the simplewave analysis to bed form migration (Appendix C), which shows that the local bed load transport rate over a bed form is proportional to the vertical distance from the trough elevation, as expressed by equation (C4). The Einstein step length formulation tells us that the local entrainment rate is a linear function of the local bed load transport rate, $q=\Lambda E$, so that the entrainment rate also increases linearly with bed elevation.

[18] Combination of equations (3) $-(7)$ and (10) yields $b_{E}=$ $a_{E} \Lambda /\left(2 \lambda_{s}\right)$, so that equations (10) and (4) yield, respectively

$$
E_{s u E}(z)=a_{E}\left(g_{d s}\left(z^{*}\right)+\Lambda /\left(2 \lambda_{s}\right)\right)
$$

$$
q_{\text {topE }}=\Lambda a_{E}
$$


where $a_{E}$ is expected to depend on the bed load transport rate, $q_{a E}$. To find out how, we rephrase the transport rate averaged over an individual bed form in equation (B12) for uniform sediment to

$$
q_{a E}=\frac{\lambda_{s}}{\lambda} \Lambda \int_{\eta_{b}^{*}}^{\eta_{t}^{*}} J(z) E_{\text {suE }}\left(z-\frac{1}{2} \eta_{\text {step }}(z)\right) d z^{*}+\frac{\lambda_{l}}{2 \lambda} q_{t o p E}
$$

where the argument equals

$$
z-\frac{1}{2} \eta_{\text {step }}(z)=\Delta f_{d s}\left[g_{d s}\left(z^{*}\right)-\Lambda /\left(2 \lambda_{s}\right)\right]+\eta_{a}
$$

Now, with equations (12), (11), and (14), and after integration over $\eta_{b}^{*}$ to $\eta_{t}^{*}$, equation (13) results in

$$
a_{E}=2 q_{a E} / \Lambda
$$

showing that for a triangular bed form the bed load transport rate over the crest is twice as large as the average bed load transport rate $\left(q_{\text {topE }}=2 q_{a E}\right)$. For reasons of simplicity, we now assume that the bed load transport rate averaged over one individual bed form is equal to the bed load transport rate averaged over the whole field of bed forms, which implies that

$$
\begin{gathered}
q_{a E}=\bar{q}_{a E} \\
a_{E}=\bar{a}_{E}=2 \bar{q}_{a E} / \Lambda
\end{gathered}
$$

[19] The set of equations is now complete. That is, for a given average bed load transport rate, $\bar{q}_{a E}$, and a given PDF of relative trough elevations, $\tilde{p}_{b E}$, we can determine the equilibrium overall entrainment and deposition densities over a series of irregular bed forms composed of uniform sediment, $\bar{E}_{e s E}, \bar{D}_{e s E}$, and $\bar{D}_{e l E}$, in equations (B5)-(B7):

$$
\begin{gathered}
\bar{E}_{e s E}(z)=\int_{\eta_{b \min }}^{\eta_{b \max }} \frac{\lambda_{s}}{\lambda} \frac{J(z)}{\Delta} E_{s u E}(z) \tilde{p}_{b E} d \eta_{b} \\
\bar{D}_{e s E}(z)=\int_{\eta_{b \min }}^{\eta_{b \max }} \frac{\lambda_{s}}{\lambda} \frac{J(z)}{\Delta} E_{s u E}\left(z-\eta_{s t e p}(z)\right) \tilde{p}_{b E} d \eta_{b} \\
\bar{D}_{\text {elE }}(z)=2 \bar{q}_{a E} \int_{\eta_{b \min }}^{\eta_{b \max }} \frac{J(z)}{\lambda \Delta} \tilde{p}_{b E} d \eta_{b}
\end{gathered}
$$

where $E_{s u E}$ is given by equation (11). The bed load transport rate, $\bar{q}_{a E}$, and the PDF of relative trough elevations, $\tilde{p}_{b E}$, need to be determined from either predictions or measurements.

\section{Tracers in Uniform Sediment}

[20] Compared to nonuniform sediment, the case of tracer particles in uniform sediment is still a straightforward one, since grain-size-selective processes do not play a role. Here the notation $f$ is used to denote the volume fraction content of tracers, with various subscripts specifying the fraction contents in question. For example, $f_{E}$ denotes the equilibrium volume fraction content of tracers at elevation $z$, and $\bar{f}_{E}$ denotes the corresponding volume fraction content averaged over a series of bed forms, i.e., the overall volume fraction content of tracers at elevation $z$. We assume that there is no difference in vertical sorting between bed forms in a series of bed forms:

$$
f_{E}(z)=\bar{f}_{E}(z)
$$

As in the analysis for uniform sediment, we assume the average bed load transport rate identical for each individual bed form. Equations (A3) and (A4) now reduce to

$$
\begin{gathered}
0=\bar{D}_{\text {eisE }}(z)-\bar{E}_{\text {eisE }}(z)+\bar{D}_{\text {eilE }}(z) \\
0=\sum_{i}^{N}\left[\bar{D}_{\text {eisE }}(z)-\bar{E}_{\text {eisE }}(z)+\bar{D}_{\text {eilE }}(z)\right]
\end{gathered}
$$

where $N$ equals 2 and the subscript $i$ denotes one of the two sediment fractions, tracer particles and nontracer particles. These equations express that, at each bed surface elevation $z$, there is a dynamic equilibrium in both composition (i.e., the volume fraction content of tracer particles) and total amount between (1) the net entrainment flux at the stoss face, averaged over all bed forms, and (2) the deposition flux at the lee face, averaged over all bed forms. We can see that, if equation (22) is met, equation (23) is satisfied automatically. Again, we assume each bed form to have a triangular shape. Combination of equations (22) and (B5)(B7) yields

$$
\begin{aligned}
0= & \int_{\eta_{\text {bmin }}}^{\eta_{\text {bmax }}} \frac{J(z)}{\lambda \Delta}\left[\lambda_{s} E_{\text {suE }}\left(z-\eta_{\text {step }}(z)\right) \bar{f}_{E}\left(z-\eta_{\text {step }}(z)\right)\right. \\
& \left.-\lambda_{s} E_{s u E}(z) \bar{f}_{E}(z)+\bar{q}_{\text {top } E} f_{\text {leelocE }}(z)\right] \tilde{p}_{b E} d \eta_{b}
\end{aligned}
$$

Selective deposition at the bed form lee face does not occur, so that the volume fraction content of tracers in the sediment deposited at elevation $z$ at the lee face equals the volume fraction content of tracers in the lee deposit, $f_{\text {leeE }}$ :

$$
f_{\text {leelocE }}(z)=f_{\text {leelocE }}=f_{\text {leeE }}
$$

For tracers in equilibrium conditions equation (B10) yields $f_{\text {leeE }}=f_{\text {top E }}$, i.e., the volume fraction content of tracers at the bed form crest equals the volume fraction content of tracers in the lee deposit.

[21] As we consider equilibrium conditions, the elevation-specific bed composition is constant. This implies that the volume fraction content of tracers in the net entrainment flux at elevation $z$, as well as the overall volume fraction content of tracers in the sediment deposited at the lee face, $\bar{f}_{\text {leelocE }}$, must equal the volume fraction content of tracers in the bed at elevation $z, \bar{f}_{E}$ :

$$
f_{E}(z)=\bar{f}_{E}=\bar{f}_{\text {leelocE }}
$$

Since preferential deposition processes do not occur, the volume fraction content of tracer particles, $\bar{f}_{E}$, is uniform over depth within the active bed. 
[22] One method to solve for the equilibrium volume fraction content of tracers, $\bar{f}_{E}$, is to derive it from the given equilibrium volume fraction content of tracers in the bed load transport, $\bar{f}_{a E}$. Similar to the analysis for uniform sediment, for tracer particles we rephrase equation (B12) to

$$
\begin{aligned}
\bar{q}_{a E} f_{a E}= & \frac{\lambda_{s}}{\lambda} \Lambda \int_{\eta_{b}^{*}}^{\eta_{t}^{*}} J(z) E_{s u E}\left(z-\frac{1}{2} \eta_{\text {step }}(z)\right) \bar{f}_{E} d z^{*} \\
& +\frac{\lambda_{l}}{2 \lambda} \bar{q}_{t o p E} \bar{f}_{t o p E}
\end{aligned}
$$

Furthermore, the elevation-specific entrainment rate for uniform sediment, $E_{s u E}$, in equation (11) must be valid for both the tracer and the nontracer particles. By substituting equations (12), (11), and (14) into equation (27), and integrating over $\eta_{b}^{*}$ to $\eta_{t}^{*}$, we find

$$
\begin{gathered}
\bar{q}_{a E} f_{a E}=\frac{1}{2} a_{E} \Lambda \bar{f}_{E} \\
\bar{q}_{a E}=\frac{1}{2} a_{E} \Lambda
\end{gathered}
$$

which yields

$$
\bar{f}_{E}=\bar{f}_{a E}
$$

which expresses the condition that the equilibrium volume fraction content of tracers in the bed, $\bar{f}_{E}$, equals the equilibrium volume fraction content of tracers in the bed load transport, $\bar{f}_{a E}$.

[23] Instead of deriving the equilibrium volume fraction content of tracers in the bed, $\bar{f}_{E}$, from the bed load transport composition, we can solve it from the volume fraction content of tracer particles in the bed at another point in time (i.e., the initial situation), $\bar{f}_{i n i}$. When there are no divergences in the rate and composition of the sediment transport (i.e., no spatial variation in both rate and composition), the total volume of tracers in the active bed is steady, as well as the volume_fraction content of tracers averaged over the active bed, $\overline{\bar{f}}$. The equilibrium volume fraction content of tracers in the bed, $\bar{f}_{E}$, must be identical to $\overline{\bar{f}}$, since both of them are uniform over all elevations of the active bed:

$$
\bar{f}_{E}=\overline{\bar{f}}=\frac{\int_{\eta_{m n}}^{\eta_{m x}} \bar{C}_{i n i}(z) d z}{\int_{\eta_{m n}}^{\eta_{m x}} \sum_{i}^{N} \bar{C}_{i n i}(z) d z}=\frac{\int_{\eta_{m n}}^{\eta_{m x}} \bar{P}_{\text {sini }}(z) \bar{f}_{i n i}(z) d z}{\int_{\eta_{m n}}^{\eta_{m x}} \bar{P}_{\text {sini }}(z) d z}
$$

where $\bar{C}_{i n i}$ denotes the overall concentration of tracer particles at bed elevation $z$ in the initial situation $\left(\bar{C}_{i n i}=\right.$ $\left.c_{b} \bar{P}_{\text {sini }} \bar{f}_{i n i}\right), \eta_{m n}$ and $\eta_{m x}$ denote the lower and upper levels of the active bed of either the initial or the equilibrium stage, that is, the stage in which the active bed covers the widest range of bed elevations. The porosity and thus the sediment concentration in the bed, $c_{b}$, are assumed to be uniform over depth. $\bar{P}_{\sin i}$ denotes the probability distribution of bed surface elevations, which is determined from the given PDF of relative trough elevations at the specific point in time, $\tilde{p}_{\text {bini }}$, using both equation (BP-58)

$$
\begin{aligned}
\bar{p}_{e}(z) & =\int_{\eta_{b \min }}^{\eta_{b \max }} p_{e}(z) \tilde{p}_{b} d \eta_{b} \\
& =\int_{\eta_{b \min }}^{\eta_{b \max }} \frac{J(z)}{\lambda \Delta}\left(\lambda_{s} \bar{p}_{s e}^{*}(z)+\lambda_{l}\right) \tilde{p}_{b} d \eta_{b}
\end{aligned}
$$

and equation (BP-2)

$$
\bar{P}_{s}=1-\int_{-\infty}^{z} \bar{p}_{e} d z
$$

where $J, \Delta, \lambda, \lambda_{s}$, and $\lambda_{l}$ are all related to the specific relative trough elevation, $\Delta_{b}$, which is described in Appendix D. $p_{s e}^{*}$ denotes the dimensionless PDF of bed surface elevations for the stoss side $\left(p_{s e}^{*}=\Delta p_{s e}\right)$.

[24] Thus there appear to be two methods to solve for the equilibrium volume fraction content of tracers in the bed, $\bar{f}_{E}$. One method is based on a given bed load transport composition, $\bar{f}_{a E}$, whereas the second method is based on a given profile of the volume fraction content of tracers at some point in time, $\bar{f}_{\text {ini }}(z)$. Note that the latter method may be used only if, from the moment that the bed composition is known until the moment equilibrium conditions are reached, divergences in the rate and composition of the sediment transport have been negligible.

\section{Nonuniform Sediment}

\subsection{Dynamic Equilibrium}

[25] The analyses for uniform sediment and tracer particles have served as stepping stones for the present analysis of nonuniform sediment. The main difference between tracers and nonuniform sediment is that the latter is characterized by grain-size-selective processes, such as the grain-size-selective deposition down a bed form lee face.

[26] Again we assume that the average bed load transport rate over each individual bed form is identical $\left(q_{a E}=\bar{q}_{a E}\right)$. Furthermore, we make no distinction in sorting between different bed forms within one series of bed forms, so that

$$
F_{i E}(z)=\bar{F}_{i E}(z)
$$

where $F_{i E}$ denotes the equilibrium volume fraction content of size fraction $i$ at elevation $z$ and $\bar{F}_{i E}$ denotes the equilibrium volume fraction content defined likewise, yet averaged over a series of bed forms. For equilibrium conditions equations (A3) and (A4) reduce to equations (22) and (23), wherein $N$ now denotes the number of grain size fractions. Using equations (B5)-(B7), equation (22) now yields

$$
\begin{aligned}
0= & \int_{\eta_{\text {bmin }}}^{\eta_{\text {bmax }}} \frac{J(z)}{\lambda \Delta}\left[\lambda_{s} E_{\text {siuE }}\left(z-\eta_{\text {stepi }}(z)\right) \bar{F}_{i E}\left(z-\eta_{\text {stepi }}(z)\right)\right. \\
& \left.-\lambda_{s} E_{\text {siuE }}(z) \bar{F}_{i E}(z)+q_{\text {topE }} F_{\text {leelociE }}(z)\right] \tilde{p}_{b E} d \eta_{b}
\end{aligned}
$$

Since we consider equilibrium conditions, the net effect of winnowing and infiltration of fines is negligible, as well as the settlement of particles too coarse to be transported [also see Blom et al. 2003]. This means that the overall composition of sediment deposited at the lee face at 
elevation $z$ must equal the bed composition at that same elevation:

$$
\bar{F}_{\text {leelociE }}(z)=\bar{F}_{i E}(z)
$$

The equilibrium state also requires that the volume fraction content of size fraction $i$ in the net entrainment flux at bed elevation $z$ at the stoss face equals the equilibrium bed composition at that same elevation, $\bar{F}_{i E}(z)$, which is illustrated in Figure $2 \mathrm{~b}$. Note that the composition of sediment deposited over the lee face of an individual bed form, $F_{\text {leeloci }}$, may differ from the bed composition at that same elevation $z, \bar{F}_{i E}$ (see Figure $2 \mathrm{~b}$ ).

[27] In order to find a solution to the equilibrium sorting profile for nonuniform sediment, $\bar{F}_{i E}$, we need to solve equation (35), which is satisfied when we assume that for each individual dune in a series of irregular dunes, the net entrainment flux of size fraction $i$ at bed elevation $z$ at the stoss face equals the deposition rate of that size fraction at the lee face:

$$
\begin{aligned}
\lambda_{s} E_{\text {SiuE }}(z & \left.-\eta_{\text {stepi }}(z)\right) \bar{F}_{i E}\left(z-\eta_{\text {stepi }}(z)\right) \\
& -\lambda_{s} E_{\text {siuE }}(z) \bar{F}_{i E}(z)+q_{\text {top }} F_{\text {leelociE }}(z)=0
\end{aligned}
$$

Note, however, that equation (35) might also be satisfied for different distributions of the different quantities over $z$. Yet the above assumption is made in order to find a solution to the model and to push the model forward. This is comparable to the argumentation leading to equation (3).

[28] Similar to equation (4), the grain-size-selective bed load transport rate over the crest, $q_{t o p i}$, in equation (B11) reduces to

$$
q_{\text {topiE }}=\Lambda_{i} \Theta_{i E}\left(z_{t}-\frac{1}{2} \eta_{\text {stepi }}\left(z_{t}\right)\right)
$$

where the weighted entrainment rate $E_{S i u E} \bar{F}_{i E}$ is substituted by $\Theta_{i E}$, so that equation (37) can be rephrased to

$$
\begin{aligned}
\lambda_{s} \Theta_{i E}(z & \left.-\eta_{\text {stepi }}(z)\right)-\lambda_{s} \Theta_{i E}(z) \\
& +\Lambda_{i} \omega_{i E}(z) \Theta_{i E}\left(z_{t}-\frac{1}{2} \eta_{\text {stepi }}\left(z_{t}\right)\right)=0
\end{aligned}
$$

where the arguments can be written as

$$
\begin{aligned}
& z-\eta_{\text {stepi }}(z)=\Delta f_{d s}\left[g_{d s}\left(z^{*}\right)-\Lambda_{i} / \lambda_{s}\right]+\eta_{a} \\
& z_{t}-\frac{1}{2} \eta_{\text {stepi }}\left(z_{t}\right)=\Delta f_{d s}\left[1-\Lambda_{i} /\left(2 \lambda_{s}\right)\right]+\eta_{a}
\end{aligned}
$$

In equation (39), the lee sorting function $\omega_{i}$ determines to what extent a specific size fraction that is transported over the bed form crest is deposited at a certain elevation of the lee face. The lee sorting function is given by equation (B13) in Appendix B.

[29] Analyzing equation (39) with its arguments, we find that a possible solution to the weighted entrainment rate, $\Theta_{i E}$, is a quadratic function of $g_{d s}$ :

$$
\Theta_{i E}(z)=a_{i E} g_{d s}^{2}\left(z^{*}\right)+b_{i E} g_{d s}\left(z^{*}\right)+c_{i E}
$$

Combination of equations (39)-(42) yields

$$
\begin{gathered}
b_{i E}=a_{i E}\left[2 / \delta_{i E}+\Lambda_{i} / \lambda_{s}-1\right] \\
c_{i E}=a_{i E} \Lambda_{i} /\left(2 \lambda_{s}\right)\left[2 / \delta_{i E}+\Lambda_{i} /\left(2 \lambda_{s}\right)-1\right]
\end{gathered}
$$

The local bed load transport rate of size fraction $i$ over the stoss face of an individual bed form, $q_{s i E}(z)$, equals

$$
\begin{aligned}
q_{s i E}(z) & =\Lambda_{i} \Theta_{i E}\left(z-\frac{1}{2} \eta_{\text {stepi }}(z)\right) \\
& =\Lambda_{i} a_{i E}\left[g_{d s}^{2}\left(z^{*}\right)+g_{d s}\left(z^{*}\right)\left(2 / \delta_{i E}-1\right)\right]
\end{aligned}
$$

This tells us that the bed load transport rate of size fraction $i$ over the stoss face, $q_{s i E}$, increases from 0 at the trough to $q_{\text {topiE }}$ at the bed form crest, where $q_{\text {topiE }}$ equals

$$
q_{\text {topiE }}=2 a_{i E} \Lambda_{i} / \delta_{i E}
$$

[30] In order to find out how the constant $a_{i E}$ is related to the bed load transport rate, we rephrase the bed load transport rate of size fraction $i$ in equation (B12) to

$$
q_{a i E}=\frac{\lambda_{s}}{\lambda} \Lambda_{i} \int_{\eta_{b}^{*}}^{\eta_{t}^{*}} \Theta_{i E}\left(z-\frac{1}{2} \eta_{\text {stepi }}(z)\right) d z^{*}+\frac{\lambda_{l}}{2 \lambda} q_{\text {topiE }}
$$

where the argument equals

$$
z-\frac{1}{2} \eta_{\text {stepi }}(z)=\Delta f_{d s}\left[g_{d s}\left(z^{*}\right)-\Lambda_{i} /\left(2 \lambda_{s}\right)\right]+\eta_{a}
$$

Using equations (46), (42), (43), (44), and (D2) in Appendix (D), we can elaborate equation (47) to

$$
a_{i E}=\bar{a}_{i E}=\frac{\bar{q}_{a i E}}{\Lambda_{i}}\left[\frac{1}{\delta_{i E}}-\frac{1}{6}+\frac{\Delta_{a}}{6 \lambda_{a} \tan (\nu)}\right]^{-1}
$$

[31] These formulations serve as a basis for the derivation of the equilibrium vertical sorting profile, $\bar{F}_{i E}(z)$, which will be addressed in the next two sections.

\subsection{Sorting Based on Transport Composition}

[32] Similar to the tracer analysis, one method for solving for the equilibrium sorting profile, $\bar{F}_{i E}$, is to derive it from the bed load transport composition, $\bar{F}_{a i E}$.

[33] According to equation (36), the equilibrium sorting profile, $\bar{F}_{i E}$, equals the equilibrium volume fraction content of size fraction $i$ deposited at elevation $z$ at the lee face, $\bar{F}_{\text {leelociE. }}$. The latter quantity can also be written as the amount of size fraction $i$ deposited at that elevation of the lee face divided by the total amount of sediment deposited at that elevation of the lee face:

$$
\bar{F}_{i E}(z)=\bar{F}_{\text {leelociE }}(z)=\bar{D}_{\text {eilE }}(z) / \sum_{i}^{N} \bar{D}_{\text {eilE }}(z)
$$

where $\bar{D}_{\text {eile }}$ and $F_{\text {leelocie }}$ are given by equations (B7) and (B9), respectively:

$$
\begin{gathered}
\bar{D}_{\text {eilE }}(z)=\bar{q}_{\text {topE }} \int_{\eta_{\text {bmin }}}^{\eta_{\text {bmax }}} \frac{J(z)}{\lambda \Delta} F_{\text {leelociE }}(z) \tilde{p}_{b E} d \eta_{b} \\
F_{\text {leelociE }}(z)=\bar{F}_{\text {leeiE }} \omega_{\text {iE }}(z)=\bar{F}_{\text {topiE }} \omega_{i E}(z)
\end{gathered}
$$


so that equation (50) can be rephrased to

$$
\bar{F}_{i E}(z)=\frac{\bar{F}_{\text {topiE }} \int_{\eta_{b \min }}^{\eta_{b \max }} \frac{J(z)}{\lambda \Delta} \omega_{i E}(z) \tilde{p}_{b E} d \eta_{b}}{\int_{\eta_{b \min }}^{\eta_{b \max }} \frac{J(z)}{\lambda \Delta} \tilde{p}_{b E} d \eta_{b}}
$$

where $\bar{F}_{\text {topiE }}\left(=\bar{q}_{\text {topiE }} / \bar{q}_{\text {topE }}\right)$ can be derived from equations (46) and (49):

$$
\bar{F}_{\text {topiE }}=\frac{\bar{F}_{a i E}\left(1-\frac{\delta_{i E}}{6}+\frac{\delta_{i E} \Delta_{a}}{6 \lambda_{a} \tan (v)}\right)^{-1}}{\sum_{i}^{N}\left[\bar{F}_{a i E}\left(1-\frac{\delta_{i E}}{6}+\frac{\delta_{i E} \Delta_{a}}{6 \lambda_{a} \tan (v)}\right)^{-1}\right]}
$$

In equation (53), the lee sorting function $\omega_{i E}$ determines the grain-size-selective sorting over the lee face of an individual dune and is given by equation (B13):

$$
\omega_{i E}(z)=J(z)\left(1+\delta_{i E} z^{*}\right)
$$

where the lee sorting parameter $\delta_{i E}$ is given by

$$
\delta_{i E}=-\gamma \frac{\bar{\phi}_{m l e e E}-\phi_{i}}{\bar{\sigma}_{a E}}\left(\bar{\tau}_{b E}\right)^{-\kappa}
$$

and the dimensionless bed shear stress averaged over the bed form length, $\bar{\tau}{ }_{b E}$, is defined as

$$
\bar{\tau}{ }_{b E}=\bar{\tau}_{b E} /\left[\left(\rho_{s}-\rho\right) g \bar{d}_{m l e e E}\right]
$$

and the geometric mean grain size of the lee deposit, $\bar{d}_{\text {mleeE }}$, as

$$
\bar{d}_{\text {mleeE }}=1 / 10002^{-\bar{\phi}_{\text {mleeE }}}
$$

The equilibrium bed shear stress, $\bar{\tau}_{b E}$, is known from either measurements or predictions, and $\gamma$ and $\kappa$ are constants that will be used as calibration coefficients in section 7 . The geometric mean grain size of the lee deposit on $\phi$ scale, $\bar{\phi}_{\text {mleeE }}$, and the arithmetic standard deviation, $\bar{\sigma}_{a E}$, are given by

$$
\begin{gathered}
\bar{\phi}_{\text {mleeE }}=\sum_{i}^{N} \phi_{i} \bar{F}_{\text {leeiE }} \\
\bar{\sigma}_{a E}^{2}=\sum_{i}^{N}\left(\phi_{i}-\bar{\phi}_{\text {mleeE }}\right)^{2} \bar{F}_{\text {leeiE }}
\end{gathered}
$$

Since the geometric mean grain size of the lee deposit, $\bar{\phi}_{\text {mleeE}}$, and the arithmetic standard deviation, $\bar{\sigma}_{a E}$, depend on the composition of the lee deposit, $\bar{F}_{\text {leeiE }}$, the equilibrium sorting profile, $\bar{F}_{i E}$, in equation (53) needs to be solved iteratively.

[34] The parameter $F_{\text {leeloci }}$ needs to fulfill two constraints, i.e., $\sum_{i}^{N} F_{\text {leeloci }}=1$ and $0 \leq F_{\text {leeloci }} \leq 1$, which express that at each bed elevation the volume fraction content summed over all size fractions must equal unity and the volume fraction content of each size fraction must be larger than or equal to zero and smaller than or equal to unity. Using the above formulations, we find that the first constraint is satisfied automatically. The second constraint can be written as

$$
\begin{gathered}
-2 \leq \delta_{i E} \leq 2 \\
-2\left(1 / \bar{F}_{\text {leeiE }}-1\right) \leq \delta_{\text {iE }} \leq 2\left(1 / \bar{F}_{\text {leeiE }}-1\right)
\end{gathered}
$$

These constraints need to be taken into account when solving for the equilibrium sorting profile.

[35] Thus equation (53) solves for the equilibrium sorting profile, $\bar{F}_{i E}$, from the volume fraction content of size fractions in the bed load transport, $\bar{F}_{a i E}$, the PDF of relative trough elevations, $\tilde{p}_{b E}$, and the lee sorting function, $\omega_{i E}$. Note that the equilibrium sorting profile is affected neither by the total bed load transport rate, $\bar{q}_{a E}$, nor by the dimensionless step length, $\alpha$.

\subsection{Sorting Based on Initial Sorting Profile}

[36] Instead of deriving the equilibrium sorting profile, $\bar{F}_{i E}$, from the bed load transport composition, $\bar{F}_{a i E}$, we can solve it from a given vertical sorting profile at another point in time, $\overline{\bar{F}}_{\text {inii, }}$ and its accompanying PDF of relative trough elevations, $\tilde{p}_{\text {bini }}$.

[37] Note that this method may be used only if, from the moment that the bed composition is specified until equilibrium conditions are reached, divergences in the grain-sizeselective transport have been negligible (i.e., no spatial variation in both rate and composition). Consequently, the volume fraction content of size fraction $i$ averaged over the active bed, $\overline{\bar{F}}_{i}$, remains constant:

$$
\overline{\bar{F}}_{i}=\frac{\int_{\eta_{m n}}^{\eta_{m x}} \bar{C}_{i}(z) d z}{\int_{\eta_{m n}}^{\eta_{m x}} \sum_{i}^{N} \bar{C}_{i}(z) d z}=\frac{\int_{\eta_{m n}}^{\eta_{m x}} \bar{P}_{s}(z) \bar{F}_{i}(z) d z}{\int_{\eta_{m n}}^{\eta_{m x}} \bar{P}_{s}(z) d z}
$$

Thus the volume fraction content of size fraction $i$ averaged over the active bed, $\overline{\bar{F}}_{i}$, must be identical at both times, so that $\overline{\bar{F}}_{i E}=\overline{\bar{F}}_{\text {inii }}$ :

$$
\frac{\int_{\eta_{m n}}^{\eta_{m x}} \bar{P}_{s}(z) \bar{F}_{i E}(z) d z}{\int_{\eta_{m n}}^{\eta_{m x}} \bar{P}_{s}(z) d z}=\frac{\int_{\eta_{m n}}^{\eta_{m x}} \bar{P}_{\text {sini }}(z) \bar{F}_{\text {inii }}(z) d z}{\int_{\eta_{m n}}^{\eta_{m x}} \bar{P}_{\text {sini }}(z) d z}
$$

from which $\bar{F}_{i E}$ can be determined. The constraints in equations (61) and (62) must be satisfied for this solution method, as well.

\section{Experimental Data}

[38] For calibrating the equilibrium sorting model, we will consider the equilibrium phases of experiments B2 and A2 conducted by Blom et al. [2003], and for verifying it those of experiments E3 and E7 conducted by Ribberink [1987]. Both sets of experiments were conducted under similar circumstances: uniform flow conditions were maintained $(\partial / \partial x=0)$ and the sediment was recirculated in order to avoid net aggradation or degradation $\left(\partial \bar{\eta}_{a} / \partial t=0\right)$. The bed was covered by bed forms. 
Table 1. Experimental Data Used as Input to the Equilibrium Sorting Model ${ }^{\mathrm{a}}$

\begin{tabular}{cccccccccc}
\hline & $\phi_{1}$ & $\phi_{2}$ & $\phi_{3}$ & $\bar{F}_{a 1}$ & $\bar{F}_{a 2}$ & $\bar{F}_{a 3}$ & $\bar{\tau}_{b}, \mathrm{~N} / \mathrm{m}^{2}$ & $\Delta_{a}, \mathrm{~cm}$ & $\lambda_{a}, \mathrm{~m}$ \\
\hline B2 & 0.56 & -1.07 & -2.51 & 0.90 & 0.05 & 0.05 & 7.4 & 12.2 & 1.79 \\
A2 & 0.56 & -1.07 & -2.51 & 0.38 & 0.38 & 0.24 & 4.6 & 4.9 & 1.38 \\
E3 & 0.36 & -0.37 & & 0.50 & 0.50 & & 2.0 & 4.4 & 0.91 \\
E7 & 0.36 & -0.37 & & 0.80 & 0.20 & & 1.4 & 4.7 & 1.10 \\
\hline
\end{tabular}

${ }^{\mathrm{a}}$ Data experiments B2 and A2 are from Blom et al. [2003]; E3 and E7 are from Ribberink [1987].

[39] Blom et al. [2003] used a sediment mixture composed of three well-sorted size fractions $\left(d_{1}=0.68 \mathrm{~mm}, d_{2}=\right.$ $2.1 \mathrm{~mm}$, and $d_{3}=5.7 \mathrm{~mm}$ ). The length, width, and height of the flume's measurement section were $50 \mathrm{~m}, 1.0 \mathrm{~m}$, and $1.0 \mathrm{~m}$ respectively. Vertical sorting profiles were measured using a new type of core sampling box. In the equilibrium phase of the experiments, about 15 box core samples were taken over the complete measurement section. The measured sorting profiles will be shown in the next sections, together with computed ones.

[40] Ribberink [1987] used a sediment mixture composed of two well-sorted sand fractions $\left(d_{1}=0.78 \mathrm{~mm}, d_{2}=\right.$ $1.29 \mathrm{~mm}$ ). The length, width, and height of the flume's measurement section were $29 \mathrm{~m}, 0.3 \mathrm{~m}$, and $0.5 \mathrm{~m}$, respectively. Bed samples were taken using sampling pipes pressed into the bed near the bed form crests. Through the sampling pipes, sand layers with a thickness of $0.5 \mathrm{~cm}$ were removed by siphoning. In experiment E3, about 50 samples were taken along the flume axis and 10 samples at the side of the flume, and in experiment E7 20 samples along the flume axis and 20 at the side.

B2

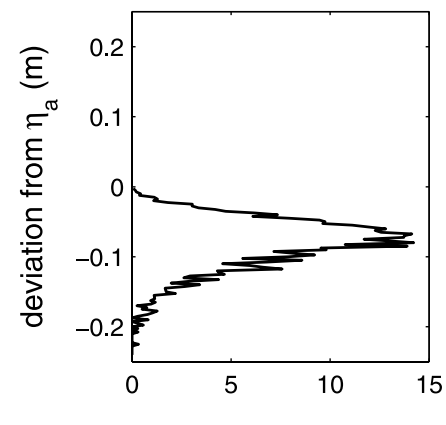

E3
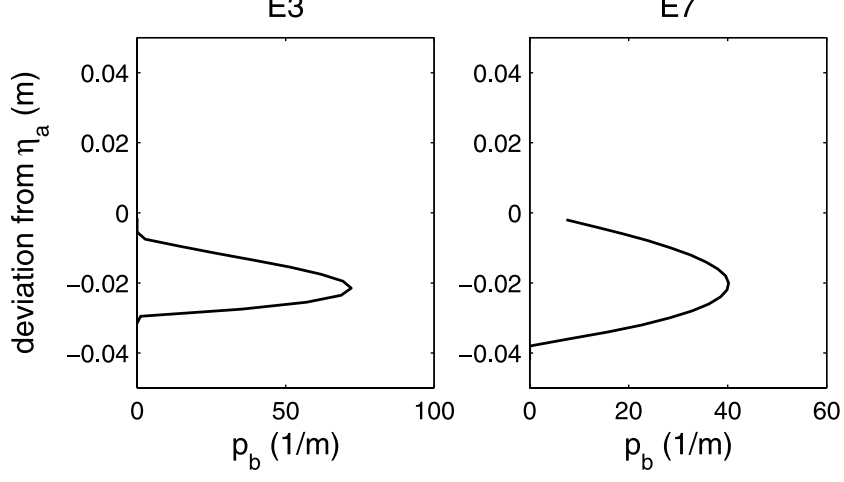

Figure 3. PDFs of trough elevations, $\tilde{p}_{b E}$, for the equilibrium phases of experiments B2, A2, E3, and E7.
Table 2. Bed Elevations Relative to the Mean Bed Level, $\bar{\eta}_{a}$, for Which the Average Error in $\bar{F}_{i E}$ is Determined ${ }^{\mathrm{a}}$

\begin{tabular}{rrrr}
\hline B2 & A2 & E3 & E7 \\
\hline 2.5 & 1.5 & 1.0 & 1.0 \\
-2.5 & 0 & 0 & 0 \\
-7.5 & -1.5 & -1.5 & -1.5 \\
-12.5 & -3.0 & -2.5 & -3.0 \\
\hline
\end{tabular}

${ }^{\mathrm{a}}$ Values are in $\mathrm{cm}$.

[41] Measured values for the bed load transport composition, $\bar{F}_{a i E}$, the average bed shear stress, $\bar{\tau}_{b E}$, the PDF of relative trough elevations, $\tilde{p}_{b E}$, and the ratio of the average bed form height to the average bed form length are used as input to the equilibrium sorting model and listed in Table 1.

[42] Trough elevations were measured in experiments A2 and B2. As trough elevations were not registered in experiments E3 and E7, the PDF of trough elevations is derived from the probability distribution of bed surface elevations, $\bar{P}_{s}$. The derivation is explained in Appendix D. Figure 3 shows the resulting PDFs of relative trough elevations for the equilibrium phases of the four experiments.

\section{Calibration}

[43] For calibrating the model, we apply the solution method based on a given bed load transport composition (section 5.2). The two constants in the lee sorting parameter, $\gamma$ and $\kappa$, are used as calibration coefficients. To find the optimum values for $\gamma$ and $\kappa$, we compare computed equilibrium sorting profiles for experiments B2 and A2 with the measured ones for a large number of given combinations of $\gamma$ and $\kappa$, and strive for the smallest difference between the measured and computed sorting profiles. To this end, we determine the average error in $\bar{F}_{i E}$, i.e., the absolute difference between the computed and measured equilibrium sorting profile, $\bar{F}_{i E}$, averaged over four bed elevations (Table 2) and over each of the size fractions.

[44] Figure 4 shows that for each experiment, for one typical value of the lee sorting parameter of the finest size fraction, $\bar{\delta}_{\text {fineE }}$, the average error in $\bar{F}_{i E}$ is minimal. The $\bar{\delta}_{i E}$ submodel in equation (56) tells us that this

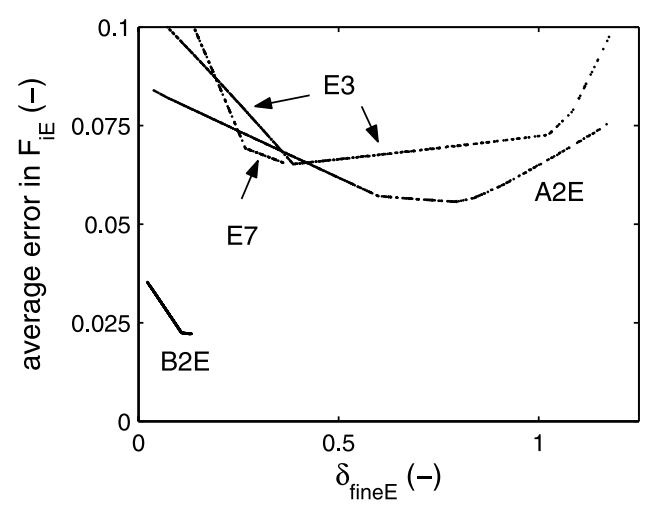

Figure 4. Lee sorting parameter for the finest size fraction, $\bar{\delta}_{\text {fineE }}$, and accompanying average error in $\bar{F}_{i E}$ for the equilibrium phases of experiments B2, A2, E3, and E7. 


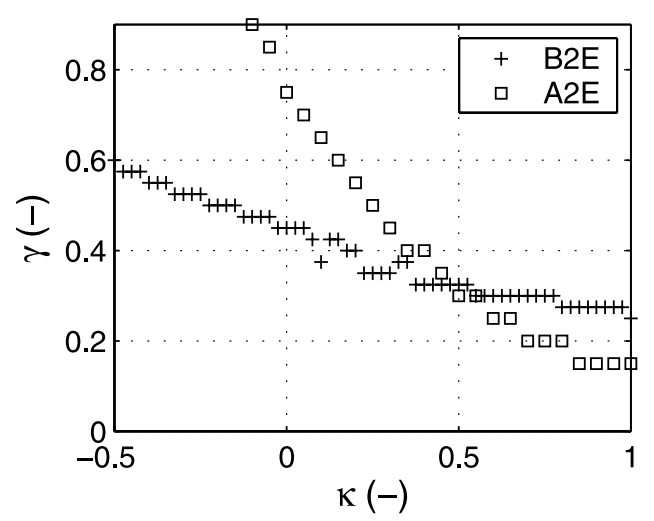

Figure 5. Combinations of $\gamma$ and $\kappa$ for which the average error in $\bar{F}_{i E}$ is minimal for the equilibrium phases of experiments $\mathrm{B} 2$ and $\mathrm{A} 2$.

optimum value for $\bar{\delta}_{\text {fineE }}$ can be found for multiple combinations of $\gamma$ and $\kappa$, which are plotted in Figure 5 for experiments $\mathrm{B} 2$ and A2. The point of intersection of the two series yields the optimum values for both $\gamma$ and $\kappa$ :

$$
\gamma=0.3 \quad \kappa=0.5
$$

Although the grain-size-selective deposition down the lee face appears primarily determined by (1) the difference in the geometric mean grain size of the lee deposit and the grain size of size fraction $i$ and (2) the arithmetic standard deviation of the mixture, the resulting value for $\kappa$ implies that the bed shear stress does affect the avalanche process, probably through the flow velocity at the bed form crest and/or the lee vortices downstream of the bed form. The sorting trend appears to become smaller with increasing bed shear stress. This was confirmed experimentally by Allen [1965] and Kleinhans [2005].

[45] For experiments B2 and A2, Figures 6 and 7 show the measured and computed equilibrium sorting profiles, using the calibrated lee sorting function. Remarkable in the measured profiles is the presence of a top layer of 1 to $2 \mathrm{~cm}$ that is significantly coarser than the material underneath. This coarse top layer seems to originate from two mechanisms. The first is the formation of a thin mobile armor layer on the stoss face, which increases the amount of exposure of the coarser grains and hinders the entrainment of fines, so as to counterbalance the higher mobility of the fines. The second mechanism concerns the deposition of sediment that was being transported over the bed form until the flow was turned off. Namely, for taking the core samples, the flow needs to be turned off, and the sediment that was being transported over the bed form settles on top of the bed form surface. At the upper elevations of the stoss face, the transported sediment is coarser than the bed form material itself, which contributes to the presence of the coarse top layer in the measured sorting profile.

[46] This coarse top layer appears to have a thickness larger than a few grain sizes. This, however, is largely due to the averaging procedure. The measured sorting profiles were determined by averaging over about 15 core samples. Since each of these elevations relative to the mean bed level was different from each other, the averaging procedure smears the coarse top layer over a larger range of bed elevations. Since in the sorting model it is assumed that the composition of the net entrainment at a certain elevation of the stoss face equals the bed composition at that elevation, this coarse top layer does not show up in the computed sorting profiles.

[47] Also noteworthy about Figures 6 and 7 is that the range of bed elevations covered by the core samples is small compared to the range covered by the computations. The measured range of bed elevations shows the range covered by the core samples, while the computed range is the range of active bed elevations. The latter is based on the PDF of measured trough elevations, as well as on the assumption that each bed form crest is located at the same vertical distance from the mean bed level as its trough (equation (D1)). In reality, however, the deepest bed form troughs are usually not accompanied by the highest crests [Leclair and Blom, 2005]. This causes the computed range of active bed elevations to be larger than the measured one. Yet since the probability density of these upper elevations being exposed to the flow, $\bar{p}_{e}$, is very small, these elevations have negligible influence on, for instance, the composition of the sediment transported over the crests.

[48] The measured sorting profile of experiment B2 suggests that the equilibrium state was not completely reached, primarily in the lower parts of the active bed (Figure 6). The lowest bed elevations covered by the core samples are characterized by a strong reduction in the proportions of the medium and coarse size fractions. Would
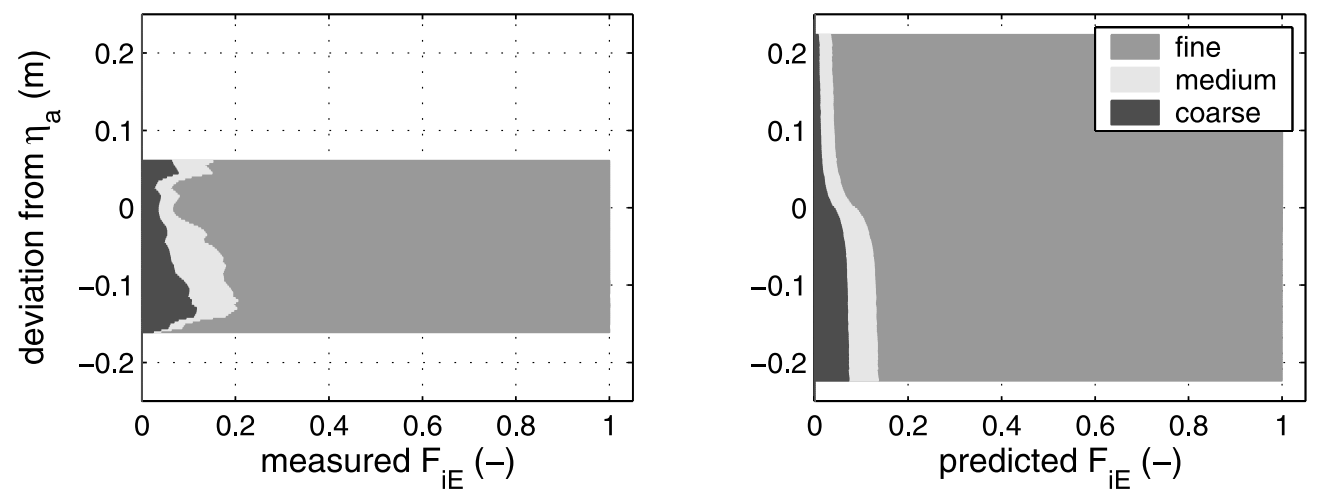

Figure 6. Measured and computed equilibrium sorting profiles for the equilibrium phase of experiment B2. 

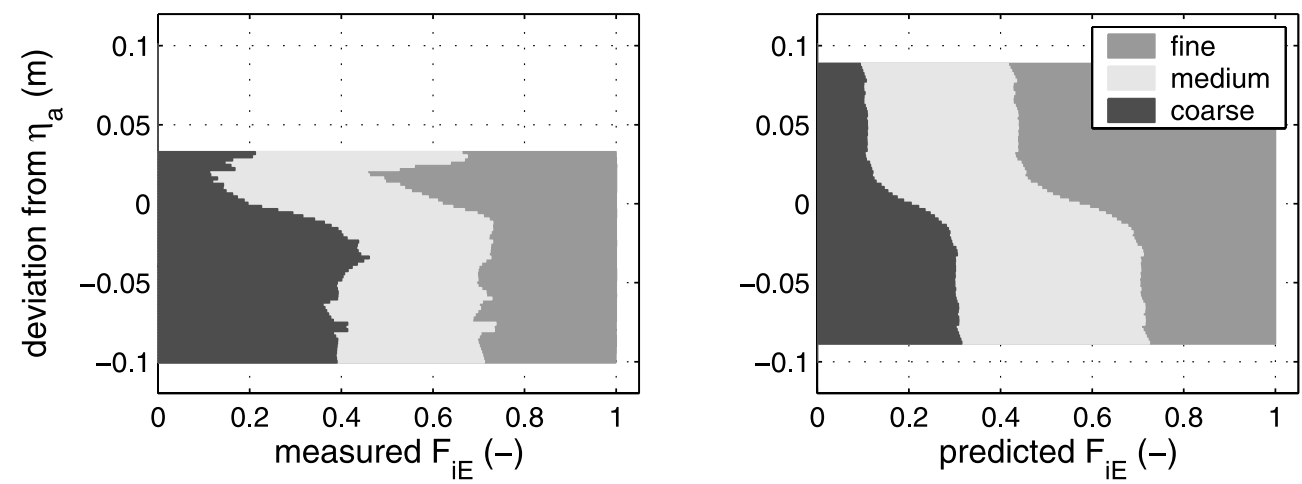

Figure 7. Measured and computed equilibrium sorting profiles for the equilibrium phase of experiment A2.

experiment B2 have been continued for a longer time, the composition at the lower elevations of the active bed would be more uniform, as suggested in the computed sorting profile.

[49] Figure 7 shows that the coarse bed layer in experiment A2 (between 2 and $4 \mathrm{~cm}$ below the mean bed level) is somewhat less distinct in the predicted sorting profile. This may suggest that in A2 the sorting profile with its coarse bed layer was not only determined by the lee face avalanche mechanism, but also by the winnowing of fines from the trough surface and subsurface [see also Blom et al., 2003, Figure 16] and the settlement of immobile coarse particles. The latter mechanisms have not been included in the present version of the model.

[50] The computed sorting profiles agree reasonably well with the measured ones. Yet the coefficients $\gamma$ and $\kappa$ were calibrated especially for these experiments, so we cannot yet draw any conclusions on the general validity of the values for $\gamma$ and $\kappa$.

\section{Verification}

[51] The equilibrium sorting model is verified by computing the equilibrium sorting profiles of the bimodal sediment experiments E3 and E7 [Ribberink, 1987]. In order to illustrate the large variation in vertical sorting over the flume, Figures 8 and 9 show the measured sorting profiles (1) averaged over samples taken along the flume axis, (2) averaged over samples taken at the sides of the flume, and (3) their average.

[52] The agreement between the measured and computed sorting profiles is very similar to experiments B2 and A2: the measured sorting profiles show a coarse top layer and the computed sorting trend over the active bed agrees well with the measured one, although the model does not reproduce the observed coarse top layer. Again, the computed range of bed elevations differs from the measured one. The measured range of bed elevations shows the range covered by the core samples, which may include inactive elevations. The computed range of bed elevations shows the range of active bed elevations, which is based on the PDF of trough elevations now derived from the PDF of measured bed elevations (computed as described in Appendix D), as well as on the assumption that each bed form crest is located at the same vertical distance from the mean bed level as its trough.
[53] In addition, the lee sorting function is tested against data on the grain-size-selective deposition down a delta lee face as measured by Kleinhans [2005] (Figure 10). A delta face is a slope at about the angle of repose over which sediment is deposited when the water depth suddenly and largely increases due to, for instance, a river flowing into a deep lake or ocean. The computed sorting profile covers the measured data reasonably well in half of the cases (N9B, N10B, and U1), but for the other cases the computed sorting is less strong than the measured one (N2A, N5B, and M1). This may be due to a tendency for the bed shear stress applied for the delta faces to be inconsistent with the one for dunes. The bed shear stress in equation (56) represents the bed shear stress averaged over a series of dunes (thus averaged over dune crests, stoss faces, and trough zones), while for the deltas we apply the bed shear stress on the delta crest. Therefore the bed shear stress for the deltas is relatively higher, resulting in a relatively smaller sorting trend. A correction factor seems necessary in order to eliminate this inconsistency. In addition, Kleinhans [2005] mentions some reasons why the sorting process down a delta face may be different from a dune. For a dune, the effects of the wake overlying the lee and the trough (i.e., the return flow and turbulence effects) are stronger than for a delta. Also, the flow over a delta crest is nearly

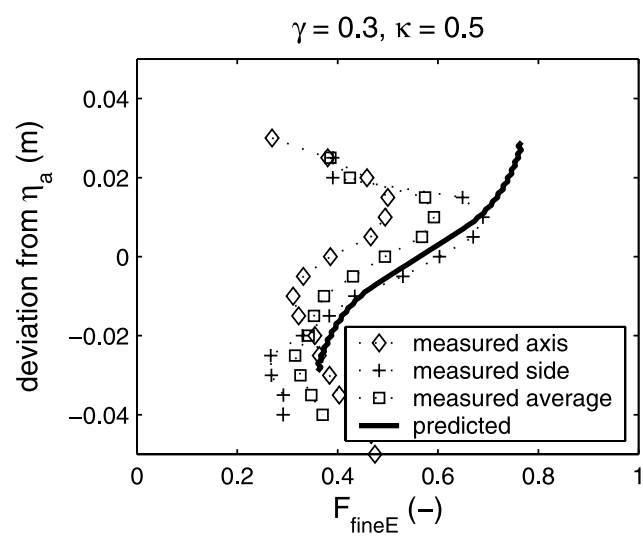

Figure 8. Measured and computed equilibrium sorting profile, $\bar{F}_{i E}$, of the fine size fraction in the bimodal mixture for the equilibrium phase of experiment E3. 


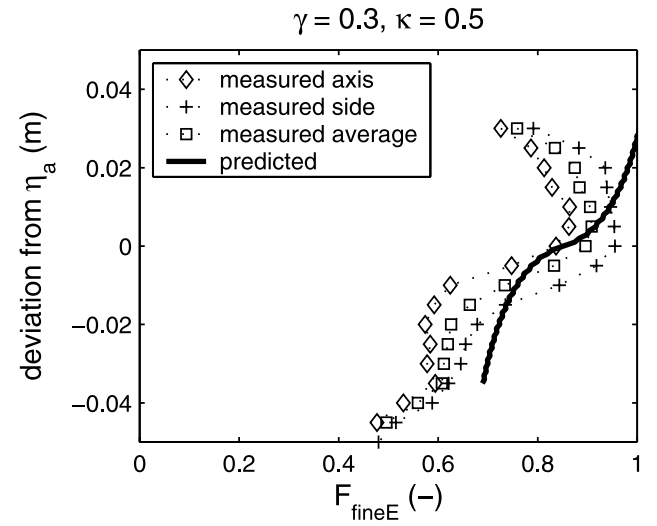

Figure 9. Measured and computed equilibrium sorting profile, $\bar{F}_{i E}$, of the fine size fraction in the bimodal mixture for the equilibrium phase of experiment E7.

uniform, whereas the flow over a dune crest is nonuniform due to flow acceleration over the stoss face.

\section{Discussion and Conclusions}

[54] We have reduced the continuum sorting model as derived by Blom and Parker [2004] to the case of equilibrium or steady conditions, resulting in an equilibrium sorting model that solves for the equilibrium vertical sorting profile of nonuniform sediment. For tracer particles, the equilibrium sorting model solves for the equilibrium volume fraction content of tracer particles in the active bed, which is uniform over all elevations of the active bed. For nonuniform sediment, the equilibrium sorting profile is mainly determined by the PDF of relative trough elevations and by the lee sorting function. It is affected neither by the average bed load transport rate, nor by the dimensionless step length. The equilibrium PDF of relative trough elevations needs to be known from either model predictions or measurements. One method for solving the equilibrium sorting profile is based on a given bed load transport composition (measured or computed), while the second is based on a given sorting profile at another point in time. By using either one of the two solution methods, we avoid introducing the uncertainties accompanying the use of a model for the grain-size-specific entrainment rate over the bed form surface. The problems accompanying such a model have been discussed by Blom and Parker [2004].

[55] The proposed framework for sediment continuity contains various submodels (e.g., leeside sorting; step length; bed form dimensions; PDF of relative trough elevations), which strongly need further development. The framework or equilibrium sorting model has been calibrated with data from two flume experiments, applying the two constants in the lee sorting function, $\gamma$ and $\kappa$, as calibration coefficients. Note that the present formulation for the lee
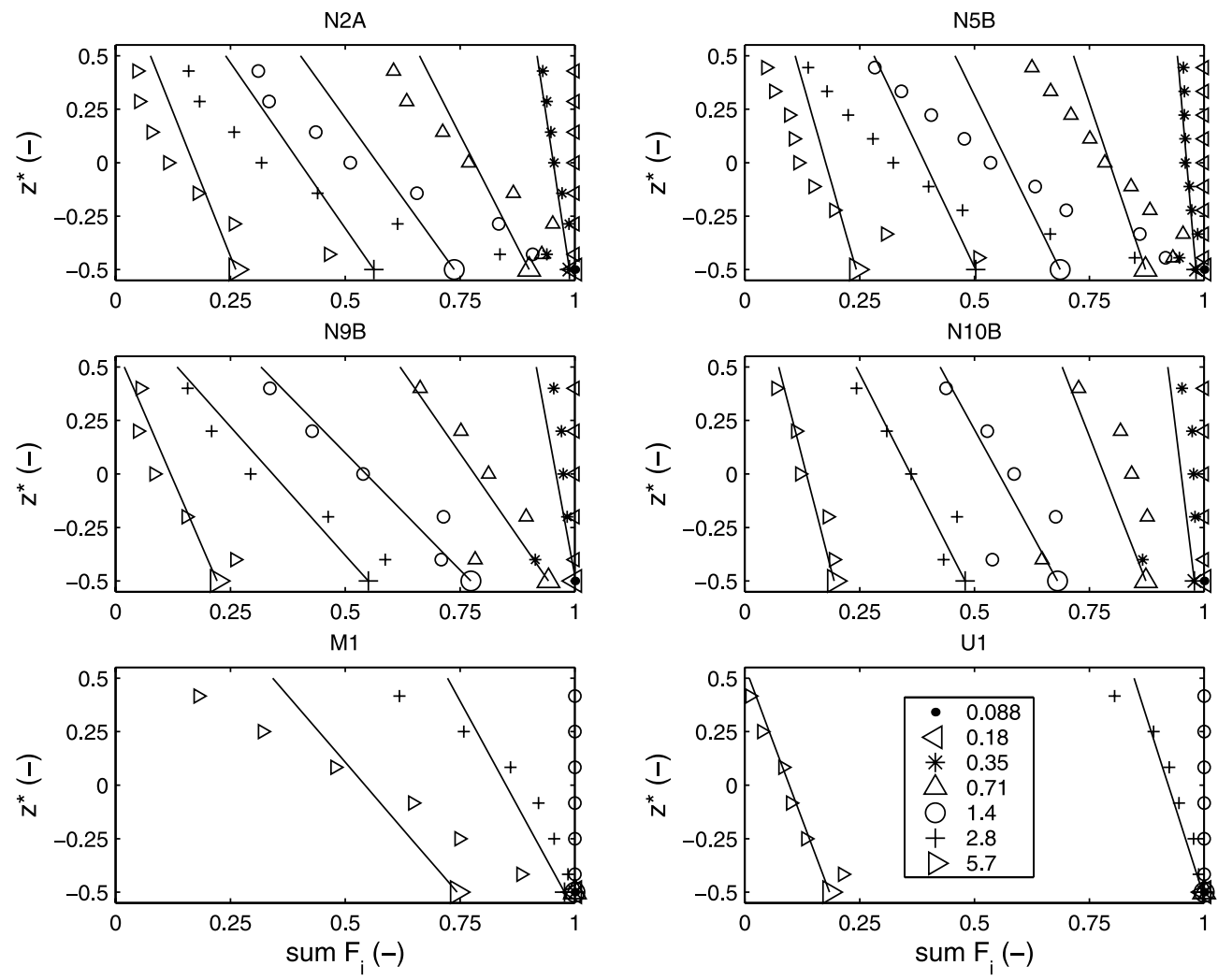

Figure 10. Measured vertical sorting profiles over delta lee faces composed of various sediment mixtures indicated by the symbols (data from Kleinhans [2005]) and computed ones indicated by the lines. The large marker at the lower limit of a line indicates the specific grain size, which is shown in the legend (in $\mathrm{mm}$ ). Note that the volume fraction content of a specific size fraction is indicated by the difference between two lines or two types of symbols. For instance, the volume fraction content of the $2.8 \mathrm{~mm}$ fraction is indicated by the difference between the crosses and the triangles pointing right. 
sorting function is introduced as a very first step toward a generic model for lee face sorting. The validity of the present formulation is limited as only two experiments were used in its calibration. Further research on the avalanche mechanism at the lee face of dunes is strongly recommended. Also, we underline the scarcity of experiments in which the effect of the variability in bed form dimensions on sediment sorting is studied. More experimental data on this subject will enable a profound calibration and validation of the present equilibrium sorting model.

[56] The order of magnitude of the computed volume fraction contents of size fractions in the active bed is reasonable. The disagreements between the measured and computed sorting profiles are small, considering (1) the simple form of the lee sorting function implemented in the continuum sorting model, (2) the approximation according to which a series of irregular bed forms are represented by a series of triangular bed forms with varying trough elevations, (3) the dispersive effects of the core sampling method [Blom et al., 2003] and the relatively small amount of bed samples taken, so that the measured equilibrium sorting profile may not be fully representative, and (4) the experiments may not have reached the equilibrium state completely, mainly in the lower part of the active bed.

[57] The winnowing of fines from the trough surface and subsurface and the settling of immobile coarse particles do seem to have a significant influence on the sorting profile. It is suggested to include these mechanisms in a future version of the model.

[58] In its present form, the new continuum sorting model incorporates bed load transport only. Suspended load transport does not seem to have played a role in experiments B2 and A2 as Blom et al. [2003] have shown that measured transport rates compare well with transport rates computed using the simple-wave approach (Appendix C). This indicates that the amount of suspended load was indeed negligible, as was the rate at which particles bypassed the lee face [Mohrig and Smith, 1996; Niño and Aracena, 1999]. Further research is required to include suspended load transport mechanisms and their effect on the resulting sorting profile.

[59] The equilibrium approach presented in this paper may be instantaneously applied in morphological models if the timescale of large-scale morphological changes is much larger than both the timescale of vertical sorting and the timescale of the evolution of the PDF of trough elevations. In a follow-up paper we will address the issue of timescales in greater detail. Clearly, we are not only interested in the vertical sorting profile in its equilibrium state, but also in the time evolution of the sorting profile. The derivation of a sorting evolution model is described by Blom [2003] and will be presented in a subsequent paper.

\section{Appendix A: PPL Framework}

[60] This section presents the main equations of the ParkerPaola-Leclair (PPL) framework [Parker et al., 2000]. Sediment conservation of size fraction $i$ at elevation $z$ is given by

$$
\frac{\partial \bar{C}_{i}}{\partial t}=c_{b} \bar{P}_{s} \frac{\partial \bar{F}_{i}}{\partial t}+c_{b} \bar{F}_{i} \frac{\partial \bar{P}_{s}}{\partial t}=\bar{D}_{e i}-\bar{E}_{e i}
$$

where $\bar{C}_{i}$ denotes the concentration of size fraction $i$ at elevation $z\left(\bar{C}_{i}=c_{b} \bar{P}_{s} \bar{F}_{i}\right), \bar{F}_{i}$ denotes the volume fraction content of size fraction $i$ at elevation $z, \bar{P}_{s}$ denotes the probability distribution of bed surface elevations indicating the probability that the bed elevation is higher than $z, \bar{D}_{e i}$ the deposition density of size fraction $i$ defined such that $\bar{D}_{e i} d x d z$ is the volume of sediment of size fraction $i$ that is deposited per unit width and time in a bed element with sides $d x$ and $d z$ at elevation $z, \bar{E}_{e i}$ the entrainment density of size fraction $i$ defined likewise, and $c_{b}$ the concentration of sediment in the bed $\left(c_{b}=1-\lambda_{b}\right.$, where $\lambda_{b}$ denotes the porosity). The bar indicates that the parameter is averaged over some horizontal distance, e.g., a large number of bed forms, $x$ denotes the horizontal coordinate on the scale of series of bed forms, $z$ denotes the vertical coordinate, and $t$ denotes the time coordinate.

[61] Applying a coordinate transformation $(\tilde{x}=x, \tilde{t}=t$, and $\tilde{z}=z-\bar{\eta}_{a}$ wherein $\tilde{z}$ denotes the deviation from the mean bed level, $\bar{\eta}_{a}$ ), and the chain rule yields

$$
\frac{\partial \bar{P}_{s}}{\partial t}=\frac{\partial \tilde{P}_{s}}{\partial t}+\bar{p}_{e} \frac{\partial \bar{\eta}_{a}}{\partial t}
$$

where $\tilde{P}_{S}$ denotes the probability distribution of bed surface elevations relative to the mean bed level, $\bar{\eta}_{a}$. The PDF of bed surface elevations, $\bar{p}_{e}$, expresses the probability density that the bed surface elevation equals $z$ or the likelihood of elevation $z$ being exposed to the flow $\left(\bar{p}_{e}=-\partial \bar{P}_{s} / \partial z=\right.$ $-\partial \tilde{P}_{S} / \partial \tilde{z}$ ). With equation (A2), equation (A1) becomes

$$
c_{b} \bar{P}_{s} \frac{\partial \bar{F}_{i}}{\partial t}+c_{b} \bar{F}_{i} \frac{\partial \tilde{P}_{s}}{\partial t}+c_{b} \bar{F}_{i} \bar{p}_{e} \frac{\partial \bar{\eta}_{a}}{\partial t}=\bar{D}_{e i}-\bar{E}_{e i}
$$

Adding up equation (A3) over all grain sizes yields

$$
c_{b} \frac{\partial \tilde{P}_{s}}{\partial t}+c_{b} \bar{p}_{e} \frac{\partial \bar{\eta}_{a}}{\partial t}=\bar{D}_{e}-\bar{E}_{e}
$$

where $\bar{D}_{e}$ denotes the deposition density defined such that $\bar{D}_{e} d x d z$ is the volume of all size fractions deposited in a bed element with sides $d x$ and $d z$ at elevation $z$ per unit width and time $\left(\bar{D}_{e}=\sum_{i}^{N} \bar{D}_{e i}\right.$ where $N$ denotes the total number of size fractions) and $\bar{E}_{e}$ the entrainment density defined likewise.

[62] Integration of equation (A4) over all bed elevations yields

$$
c_{b} \frac{\partial \bar{\eta}_{a}}{\partial t}=\bar{D}-\bar{E}\left(=-\frac{\partial \bar{q}_{a}}{\partial x}\right)
$$

where $\bar{D}$ denotes the volume of all size fractions deposited per unit area and time $\left(\bar{D}=\int_{-\infty}^{\infty} \bar{D}_{e} d z\right)$, and $\bar{E}$ the entrainment rate defined likewise. In equation (A5) we recognize the standard sediment continuity equation, where $\bar{q}_{a}$ denotes the bed load transport rate averaged over a series of bed forms.

\section{Appendix B: BP Framework}

[63] The work by Blom and Parker [2004] adapts the derivation of formulations for the grain-size-specific and 
bed-elevation-specific entrainment and deposition fluxes as required for the PPL framework to the case of a field of dunes. This section presents the fundamental equations of the Blom-Parker framework. They distinguish between an entrainment flux and a deposition flux at the stoss face, $\bar{E}_{\text {eis }}$ and $\bar{D}_{\text {eis }}$, and a deposition flux at the lee face, $\bar{D}_{\text {eil }}$, and rewrite the right-hand terms of equation (A3):

$$
c_{b} \bar{P}_{s} \frac{\partial \bar{F}_{i}}{\partial t}+c_{b} \bar{F}_{i} \frac{\partial \tilde{P}_{s}}{\partial t}+c_{b} \bar{F}_{i} \bar{p}_{e} \frac{\partial \bar{\eta}_{a}}{\partial t}=\bar{D}_{e i s}-\bar{E}_{e i s}+\bar{D}_{e i l}
$$

The parameter $E_{\text {siu }}$ is introduced as the volume of sediment of size fraction $i$ picked up from the bed per unit length, width, and time, in case only size fraction $i$ is present. The weighted entrainment rate $E_{s i}(x)$ denotes the volume of sediment of grain size $d_{i}$ locally entrained from the stoss face per unit area and time:

$$
E_{s i}(x)=E_{\text {siu }}(x) F_{i}(x)
$$

and the weighted deposition rate $D_{s i}$ of size fraction $i$ at $x$ equals the weighted entrainment rate of this size fraction one step length upstream of $\left.x\left(D_{s i}(x)=E_{s i}\left(x-\Lambda_{i}\right)\right)\right)$ :

$$
D_{s i}(x)=E_{s i u}\left(x-\Lambda_{i}\right) F_{i}\left(x-\Lambda_{i}\right)
$$

where the Einstein step length is given by $\Lambda_{i}=\alpha d_{i}$. The transport rate of size fraction $i$ at coordinate $x$ at the stoss face, $q_{s i}$, is given by

$$
q_{s i}(x)=\int_{0}^{\Lambda_{i}} E_{s i u}(x-y) F_{i}(x-y) d y
$$

The total volume transport rate per unit width at $x$ is denoted as $q_{s}(x)$, where $q_{s}(x)=\sum_{i}^{N} q_{s i}(x)$. The volume fraction content of size fraction $i$ in the transported sediment on the stoss face, $F_{q s i}$, then equals $q_{s i} / q_{s}$. For the derivation of these equations we refer to the derivation of equations (BP-15), (BP-16), and (BP-19) by Blom and Parker [2004].

[64] Blom and Parker [2004] derive the following expressions for the entrainment and deposition densities averaged over a series of irregular bed forms:

$$
\begin{gathered}
\bar{E}_{\text {eis }}(z)=\int_{\eta_{\text {bmin }}}^{\eta_{\text {mmax }}} \frac{\lambda_{s}}{\lambda} p_{\text {se }}(z) E_{\text {siu }}(z) \bar{F}_{i}(z) \tilde{p}_{b} d \eta_{b} \\
\bar{D}_{\text {eis }}(z)=\int_{\eta_{\text {bmin }}}^{\eta_{\text {bmax }}} \frac{\lambda_{s}}{\lambda} p_{\text {se }}(z) E_{\text {siu }}\left(z-\eta_{\text {stepi }}(z)\right) \bar{F}_{i}\left(z-\eta_{\text {stepi }}(z)\right) \\
\cdot \tilde{p}_{b} d \eta_{b} \\
\bar{D}_{\text {eil }}(z)=\int_{\eta_{\text {bmin }}}^{\eta_{\text {bmax }}} \frac{\lambda_{l}}{\lambda} p_{\text {le }}(z) D_{l} F_{\text {leeloci }}(z) \tilde{p}_{b} d \eta_{b}
\end{gathered}
$$

where the subscript $s$ indicates the stoss face, the subscript $l$ indicates the lee face, $\eta_{\text {stepi }}$ denotes the vertical step length at elevation $z$ on the stoss face for size fraction $i, D_{l}$ denotes the deposition rate at the lee face, $F_{\text {leeloci }}$ denotes the volume fraction content of size fraction $i$ in the sediment deposited at elevation $z$ at the lee face, $\tilde{p}_{b}$ denotes the PDF of trough elevations relative to the mean bed level for a series of bed forms, indicating the probability density that the trough elevation equals $z$, weighted by the horizontal distance involved, $\eta_{b}$ denotes the trough elevation, $\eta_{b \max }$ denotes the highest trough elevation, and $\eta_{b \min }$ denotes the lowest trough elevation. Note that the integral in these equations denotes the procedure of averaging over all trough elevations. For the derivation of these equations we refer to equations (BP-59)-(BP-61) of Blom and Parker [2004].

[65] The deposition rate at the lee face, $D_{l}$, the volume fraction content of size fraction $i$ in the sediment deposited at the lee face, $F_{\text {leeloci }}$, and the volume fraction content of size fraction $i$ in the deposit at the bed form lee face, $F_{l e e i}$, are given by equations (BP-28), (BP-36), and (BP-31):

$$
\begin{gathered}
D_{l}=\frac{q_{\text {top }}}{\lambda_{l}}-\frac{\lambda}{\lambda_{l}} \frac{\partial q_{a}}{\partial x} \\
F_{\text {leeloci }}=F_{\text {leei }} \omega_{i} \\
F_{\text {leei }}=\frac{1}{\lambda_{l} D_{l}}\left(q_{\text {topi }}-\lambda \frac{\partial q_{a i}}{\partial x}\right)
\end{gathered}
$$

where the bed load transport rate of size fraction $i$ at the bed form crest, $q_{\text {topi }}$, and the bed-form-averaged bed load transport rate of size fraction $i, q_{a i}$, are given by equations (BP-B16) and (BP-B17):

$$
q_{\text {topi }}=\lambda_{s} \int_{\eta_{t}-\eta_{\text {stepi }}}^{\eta_{t}} E_{\text {siu }}(z) F_{i}(z) p_{s e}(z) d z
$$

$$
\begin{aligned}
q_{a i}= & \frac{\lambda_{s}^{2}}{\lambda} \int_{\eta_{b}}^{\eta_{t}} \int_{0}^{\eta_{\text {stepi }}} E_{\text {siu }}\left(z-z^{\prime}\right) F_{i}\left(z-z^{\prime}\right) p_{\text {se }}(z) p_{\text {se }}\left(z^{\prime}\right) d z^{\prime} d z \\
& +\frac{\lambda_{l}}{2 \lambda} D_{l} \lambda_{l} F_{\text {leei }}
\end{aligned}
$$

where the bed-form-averaged bed load transport rate is given by $q_{a}=\sum_{i}^{N} q_{a i}$ and the bed-form-averaged volume fraction content of size fraction $i$ in the bed load transport $F_{a i}=q_{a i} / q_{a}$. The parameters $q_{t o p}$ and $F_{\text {topi }}$, indicating the bed load transport at the bed form crest, are defined likewise. The lee sorting function, $\omega_{i}$, determines to what extent a specific size fraction that is transported over the bed form crest is deposited at a certain elevation of the lee face, and is given by equation (BP-38):

$$
\omega_{i}=J\left(1+\delta_{i} \hat{z}^{*}\right)
$$

The Heaviside function $J(z)$ equals 1 when considering an elevation covered by the specific bed form. For triangular dunes $\hat{z}^{*}=z^{*}=\left(z-\eta_{a}\right) / \Delta$. As a very first step toward a generic formulation, the following expression is proposed for the lee sorting parameter $\delta_{i}$ :

$$
\delta_{i}=-\gamma \frac{\phi_{m l e e}-\phi_{i}}{\sigma_{a}}(\tau *)^{-\kappa}
$$

also see equation (BP-40). Herein $\phi_{\text {mlee }}$ denotes the geometric mean grain size of the lee deposit on $\phi$-scale, $\phi_{i}$ denotes the grain size of size fraction $i$ on $\phi$ scale $\left(\phi_{i}=\right.$ ${ }^{2} \log 1000 d_{i}$ with $d_{i}$ in meters), and $\tau$ 娄 denotes the dimensionless bed shear stress averaged over the bed form length. The constant $\gamma$ weights the relative importance of the grain size term on the right-hand side of (B14), while the value of $\kappa$ sets the relative importance of the dimensionless 
bed shear stress term. For the derivation of the above equations we refer to Blom and Parker [2004].

\section{Appendix C: Simple-Wave Approach}

[66] Bagnold [1941] derived a formulation for the spatial variation of the total bed load transport rate over a bed form by applying the simple-wave equation to bed form migration, assuming bed forms to be closed units without any bed load transport between them. The bed forms may have an arbitrary shape, but this shape remains invariant. Local aggradation and degradation along a bed form is described by the sediment continuity equation:

$$
c_{b} \frac{\partial \eta}{\partial t}=-\frac{\partial q}{\partial x}
$$

Assuming no deformation of the bed form, a simple-wave equation is used to describe its migration:

$$
\frac{\partial \eta}{\partial t}+c \frac{\partial \eta}{\partial x}=0
$$

where $c$ denotes the migration speed of the bed form. Combination of equations (C1) and (C2) yields

$$
\frac{\partial q}{\partial x}-c c_{b} \frac{\partial \eta}{\partial x}=0
$$

Integration of equation (C3) over $x$ leads to $q=c c_{b} \eta+q_{0}$, where $q_{0}$ is an integration constant. In the troughs the bed load transport rate is assumed to equal zero, so that

$$
q=c c_{b}\left(\eta-\eta_{b}\right)
$$

which for a bed form crest yields $c=q_{\text {top }} /\left(c_{b} \Delta\right)$. The bedform-averaged bed load transport rate equals

$$
q_{a}=\frac{1}{\lambda} \int_{0}^{\lambda} q d x=c c_{b} \beta \Delta
$$

where $\beta$ denotes the bed form shape factor, which is defined such that the mean bed level $\eta_{a}$ is located at $\beta \Delta$ above the trough elevation $\eta_{b}\left(\beta=\left(\eta_{a}-\eta_{b}\right) / \Delta\right)$, so that for triangular bed forms $\beta=\frac{1}{2}$.

\section{Appendix D: PDF of Trough Elevations}

[67] The geometrical properties of the individual triangular dunes are described by the following simple rules. Each crest is assumed to have the same absolute distance to the mean bed level as its trough, and the steepness of the lee faces is assumed to equal the angle of repose $(\nu)$. The dune length is assumed to be proportional to the bed form height and the ratio of the average dune length $\lambda_{a}$ to the average dune height $\Delta_{a}$ :

$$
\begin{gathered}
\Delta=2 \Delta_{b} \\
\lambda=\left(\lambda_{a} / \Delta_{a}\right) \Delta \\
\lambda_{l}=\Delta / \tan (\nu) \\
\lambda_{s}=\lambda-\lambda_{l}
\end{gathered}
$$

see also equations $(\mathrm{BP}-50)-(\mathrm{BP}-53)$ and Figure 1 . Note that these simple geometric relations are not supposed to be generally valid and their applicability should be checked against data when applying them.

[68] Equation (32) shows how the overall PDF of bed surface elevations, $\bar{p}_{e}$, is related to the dimensionless PDF of bed surface elevations for the stoss side, $\bar{p}_{s e}^{*}$, and the PDF of relative trough elevations, $\tilde{p}_{b}$. For triangular dunes equation (32) reduces to

$$
\bar{p}_{e}(z)=\int_{\eta_{b \min }}^{\eta_{b \max }} \frac{J(z)}{\Delta} \tilde{p}_{b} d \eta_{b}
$$

Transformation of equation (D5) shows how we can determine the PDF of relative trough elevations, $\tilde{p}_{b}$, from the PDF of bed surface elevations, $\bar{p}_{e}$ :

$$
\tilde{p}_{b}(z)= \begin{cases}0 & \text { if } z>\bar{\eta}_{a} \\ \Delta(z) \partial \bar{p}_{e}(z) / \partial z & \text { if } z \leq \bar{\eta}_{a}\end{cases}
$$

Note that in equation (D6) the bed form height, $\Delta$, is dependent on the relative trough elevation, $\Delta_{b}$, whence $\Delta$ is dependent on bed elevation $z$ :

$$
\Delta(z)=2 \Delta_{b}=2\left(\bar{\eta}_{a}-z\right)
$$

using equation (D1). Also note that in order to use equation (D6) the overall PDF of trough elevations, $\bar{p}_{e}$, must be monotonic in $z$ up to the mean bed level, $\bar{\eta}_{a}$, i.e., $\bar{p}_{e}$ must increase with $z$ up to the mean bed level. This constraint is satisfied for a series of triangular dunes for which the mean bed level of all individual bed forms is located at the same bed elevation.

\section{Notation}

$a_{i E}$ grain-size-specific constant in $\Theta_{i E}$

$b_{i E}$ grain-size-specific constant in $\Theta_{i E}$.

$c$ bed form migration speed, $\mathrm{m} \mathrm{s}^{-1}$.

$c_{b}$ sediment concentration within the bed $\left(c_{b}=\right.$ $\left.1-\lambda_{b}\right)$

$c_{i E} \quad$ grain-size-specific constant in $\Theta_{i E}$.

$\bar{C}_{i}$ concentration of size fraction $i$ at elevation $z$, averaged over a series of bed forms.

$d_{i}$ grain size of size fraction $i, \mathrm{~m}$.

$d_{\text {mlee }}$ geometric mean grain size of the lee deposit, $\mathrm{m}$.

$\bar{D}$ volume of deposited sediment per unit area and time, summed over all size fractions and averaged over a series of bed forms, $\mathrm{m} \mathrm{s}^{-1}$.

$\bar{D}_{e}$ deposition density defined like $\bar{D}_{e i}$ but summed over all size fractions, $\mathrm{s}^{-1}$.

$\bar{D}_{e i}$ deposition density of size fraction $i$ defined such that $\bar{D}_{e i} d x d z$ is the volume of size fraction $i$ deposited in a bed element with sides $d x$ and $d z$ at elevation $z$, per unit width and time, averaged over a series of bed forms, $\mathrm{s}^{-1}$.

$D_{l}$ deposition rate at the lee face, $\mathrm{m} \mathrm{s}^{-1}$.

$D_{s i}$ volume of size fraction $i$ locally deposited onto the stoss face, per unit area and time, $\mathrm{m} \mathrm{s}^{-1}$. 
$\bar{E}$ volume of entrained sediment defined like $\bar{D}$, $\mathrm{m} \mathrm{s}^{-1}$.

$\bar{E}_{e} \quad$ entrainment density defined like $\bar{D}_{e}, \mathrm{~s}^{-1}$.

$\bar{E}_{e i}$ entrainment density of size fraction $i$ defined like $\bar{D}_{e i}, \mathrm{~s}^{-1}$.

$E_{s i} \quad$ volume of size fraction $i$ locally entrained from the stoss face, per unit area and time, $\mathrm{m} \mathrm{s}^{-1}$.

$E_{\text {siu }} \quad$ volume of size fraction $i$ locally entrained from the stoss face, per unit area and time, if only sediment of size fraction $i$ would be present, $\mathrm{m}$ $\mathrm{s}^{-1}$.

$f$ volume fraction content of tracers.

$f_{d s}$ elevation of the stoss face as a function of its horizontal coordinate, dimensionless.

$\bar{F}_{a i}$ volume fraction content of size fraction $i$ in the bed load transport, averaged over a series of bed forms.

$\bar{F}_{i} \quad$ volume fraction content of size fraction $i$ in the bed at elevation $z$, averaged over a series of bed forms.

$\bar{F}_{\text {leei }} \quad$ volume fraction content of size fraction $i$ in the lee deposit, averaged over a series of bed forms.

$F_{\text {leeloci }}$ volume fraction content of size fraction $i$ in the sediment deposited at elevation $z$ at the lee face.

$\bar{F}_{\text {topi }}$ volume fraction content of size fraction $i$ in the bed load transport over the bed form crest, averaged over a series of bed forms.

$g$ gravitational acceleration, $\mathrm{m} \mathrm{s}^{-2}$.

$g_{d s}$ horizontal coordinate of the stoss face as a function of its elevation, dimensionless.

$J(z)$ Heaviside function which equals 1 when considering an elevation covered by the bed form.

$N$ total number of size fractions.

$\tilde{p}_{b}$ probability density function of trough elevations relative to the mean bed level for a series of bed forms, weighted by the horizontal distance involved, $\mathrm{m}^{-1}$.

$\bar{p}_{e}$ probability density function of bed surface elevations for a series of bed forms, $\mathrm{m}^{-1}$.

$\bar{p}_{e}^{*}$ probability density function of bed surface elevations for a series of bed forms, dimensionless.

$\bar{P}_{s}$ probability distribution of bed surface elevations for a series of bed forms.

$\tilde{P}_{s}$ probability distribution of bed surface elevations relative to the mean bed level.

$q$ volume of bed load transport per unit width and time (excluding pores), $\mathrm{m}^{2} \mathrm{~s}^{-1}$.

$\bar{q}_{a}$ volume of bed load transport per unit width and time (excluding pores), averaged over a series of bed forms, $\mathrm{m}^{2} \mathrm{~s}^{-1}$.

$\bar{q}_{\text {top }}$ volume of bed load transport at the bed form crest per unit width and time (excluding pores), averaged over a series of bed forms, $\mathrm{m}^{2} \mathrm{~s}^{-1}$.

$t$ time coordinate, $\mathrm{s}$.

$x$ horizontal coordinate, $\mathrm{m}$.

$x^{*}$ horizontal coordinate on the scale of an individual bed form, dimensionless. $z$ vertical coordinate, $\mathrm{m}$.

$\tilde{z}$ vertical coordinate relative to the mean bed level, $\mathrm{m}$.

$z^{*}$ vertical coordinate relative to the mean bed level, dimensionless.

$\hat{z}^{*}$ vertical coordinate relative to the mean bed level, dimensionless.

$z_{t}$ bed form crest elevation $\left(z_{t}=\eta_{t}\right), \mathrm{m}$.

$\alpha$ step length, dimensionless.

$\beta$ bed form shape factor.

$\gamma$ constant in lee sorting function.

$\delta_{i}$ lee sorting parameter.

$\Delta$ bed form height, $\mathrm{m}$.

$\Delta_{a}$ bed form height averaged over a series of bed forms, $m$.

$\Delta_{b}$ relative trough elevation $\left(\Delta_{b}=\bar{\eta}_{a}-\eta_{b}\right), \mathrm{m}$.

$\eta \quad$ local bed surface elevation, $\mathrm{m}$.

$\eta_{a}$ bed surface elevation averaged over a single bed form, $\mathrm{m}$.

$\bar{\eta}_{a}$ bed surface elevation averaged over a series of bed forms (mean bed level), m.

$\eta_{b}$ bed form trough elevation, $\mathrm{m}$.

$\eta_{b \max }$ highest bed form trough elevation, $\mathrm{m}$.

$\eta_{b \min }$ lowest bed form trough elevation, $\mathrm{m}$.

$\eta_{t}$ bed form crest elevation, $\mathrm{m}$.

$\eta_{m n} \quad$ lower limit of the active bed, $\mathrm{m}$.

$\eta_{m x}$ upper limit of the active bed, $m$.

$\eta_{\text {stepi }}$ step length in $z$ direction for size fraction $i, \mathrm{~m}$.

$\Theta_{i}$ weighted entrainment rate of size fraction $i$ $\left(\Theta_{i}=E_{\text {siu }} \bar{F}_{i}\right), \mathrm{m} \mathrm{s}^{-1}$.

$\kappa$ constant in lee sorting function.

$\lambda$ bed form length, $\mathrm{m}$.

$\lambda_{a}$ bed form length averaged over a series of bed forms, $\mathrm{m}$.

$\lambda_{b}$ porosity.

$\Lambda_{i} \quad$ step length of size fraction $i, \mathrm{~m}$.

$v$ angle of repose, degrees.

$\rho$ density of water, $\mathrm{kg} \mathrm{m}^{-3}$.

$\rho_{s}$ density of sediment, $\mathrm{kg} \mathrm{m}^{-3}$.

$\sigma_{a}$ arithmetic standard deviation of the composition of the lee deposit; since its unit equals the unit of $\phi$, which is nonsensical $\left({ }^{2} \log \mathrm{mm}\right)$, it is left out.

$\bar{\tau}_{b}$ bed shear stress averaged over a series of bed forms, $\mathrm{N} \mathrm{m}^{-2}$.

$\phi_{i} \quad$ grain size of size fraction $i$ on $\phi$ scale; since the unit of $\phi$ is nonsensical $\left({ }^{2} \log \mathrm{mm}\right)$, it is left out.

$\phi_{\text {mlee }}$ geometric mean grain size on $\phi$ scale of the lee deposit.

$\omega_{i} \quad$ lee sorting function.

Subscripts

$E$ equilibrium conditions.

$i$ number of the size fraction.

$l$ lee face.

$s$ stoss face.

$u$ case of sediment of only size fraction $i$, although hiding exposure effects may be included.

[69] An asterisk denotes a dimensionless parameter. An overbar denotes that a parameter is horizontally averaged over a series of bed forms. A double overbar indicates a parameter is horizontally averaged over a series of bed 
forms and over a specific range of bed elevations. A tilde indicates a parameter is relative to the mean bed level.

[70] Acknowledgments. The first author worked on the project in the pursuit of a doctoral degree at the Department of Civil Engineering of the University of Twente. The study was supported by the Institute for Inland Water Management and Waste Water Treatment (Rijkswaterstaat RIZA) of the Ministry of Transport, Public Works, and Water Management in the Netherlands, by WL Delft Hydraulics, and by the University of Twente. The authors thank Maarten Kleinhans for providing them with the flume data on sorting down the delta faces. The authors thank the Associate Editor, Patricia Wiberg, and the two reviewers, Stefano Lanzoni and Yarko Niño, who all greatly contributed to the readability of the manuscript. The Netherlands Organization for Scientific Research (NWO) and the Prince Bernhard Cultural Foundation are acknowledged for their financial support for a 3 month stay by the first author at the St. Anthony Falls Laboratory.

\section{References}

Allen, J. R. L. (1965), Sedimentation to the lee of small underwater sand waves: An experimental study, J. Geol., 73, 95-116.

Bagnold, R. A. (1941), The Physics of Blown Sand and Desert Dunes, Methuen, New York.

Blom, A. (2003), A vertical sorting model for rivers with non-uniform sediment and dunes, Ph.D. thesis, Univ. of Twente, Enschede, Netherlands.

Blom, A., and G. Parker (2004), Vertical sorting and the morphodynamics of bedform-dominated rivers: A modeling framework, J. Geophys. Res. 109, F02007, doi:10.1029/2003JF000069.

Blom, A., J. S. Ribberink, and H. J. de Vriend (2003), Vertical sorting in bed forms: Flume experiments with a natural and a trimodal sediment mixture, Water Resour. Res., 39(2), 1025, doi:10.1029/2001WR001088.
Einstein, H. A. (1950), The bed-load function for sediment transportation in open channel flows, U.S. Dep. Agric. Tech. Bull., 1026.

Hirano, M. (1971), River bed degradation with armouring, Trans. Jpn. Soc. Civ. Eng., 3(2), 194-195.

Kleinhans, M. G. (2005), Grain-size sorting in grainflows at the lee side of deltas, Sedimentology, 52, 291-311, doi:10.1111/j.13653091.2005.00698.x.

Leclair, S. F., and A. Blom (2005), A qualitative analysis of the distribution of bed-surface elevation and the characteristics of associated deposits for subaqueous dunes, in Fluvial Sedimentology VII, edited by M. D. Blum, S. B. Marriott, and S. F. Leclair, Spec. Publ. Int. Assoc. Sedimentol., 35, $121-134$.

Mohrig, D., and J. D. Smith (1996), Predicting the migration rates of subaqueous dunes, Water Resour. Res., 32(10), 3207-3217.

Niño, Y., and D. Aracena (1999), Experimental observations of ripple growth in non-uniform sediment, in Proceedings of IAHR Symposium on River, Coastal and Estuarine Morphodynamics, edited by G. Seminara, pp. 241-250, Univ. of Genova, Genoa, Italy.

Parker, G., C. Paola, and S. Leclair (2000), Probabilistic Exner sediment continuity equation for mixtures with no active layer, J. Hydraul. Eng., $126(11), 818-826$

Ribberink, J. S. (1987), Mathematical modelling of one-dimensional morphological changes in rivers with non-uniform sediment, Ph.D. thesis, Delft Univ., Delft, Netherlands.

A. Blom, H. J. de Vriend, and J. S. Ribberink, Water Engineering and Management, Civil Engineering, University of Twente, P.O. Box 217, 7500 AE Enschede, Netherlands. (a.blom@utwente.nl; huib.devriend@ wldelft.nl; j.s.ribberink@utwente.nl)

G. Parker, Department of Civil and Environmental Engineering and Department of Geology, University of Illinois at Urbana-Champaign, Urbana, IL 61801, USA. (parkerg@uiuc.edu) 